\title{
Global Existence and Regularity for the 3D Stochastic Primitive Equations of the Ocean and Atmosphere with Multiplicative White Noise*
}

\author{
A. Debussche ${ }^{\sharp}$, N. Glatt-Holtz ${ }^{b}$, R. Temam ${ }^{b}$ and M. Ziane \\ \#IRMAR-UMR 6625, ENS Cachan Bretagne \\ 35170 Bruz, France \\ ${ }^{b}$ The Institute for Scientific Computing and Applied Mathematics \\ Indiana University, Bloomington, IN 47405, USA \\ $\natural^{\natural}$ epartment of Mathematics, University of Southern California \\ Los Angeles, CA 90089, USA
}

May 16, 2012

\begin{abstract}
The Primitive Equations are a basic model in the study of large scale Oceanic and Atmospheric dynamics. These systems form the analytical core of the most advanced General Circulation Models. For this reason and due to their challenging nonlinear and anisotropic structure the Primitive Equations have recently received considerable attention from the mathematical community.

On the other hand, in view of the complex multi-scale nature of the earth's climate system, many uncertainties appear that should be accounted for in the basic dynamical models of atmospheric and oceanic processes. In the climate community stochastic methods have come into extensive use in this connection. For this reason there has appeared a need to further develop the foundations of nonlinear stochastic partial differential equations in connection with the Primitive Equations and more generally.

In this work we study a stochastic version of the Primitive Equations. We establish the global existence and uniqueness of strong, pathwise solutions for these equations in dimension 3 for the case of a nonlinear multiplicative noise. The proof makes use of anisotropic estimates, $L_{t}^{p} L_{x}^{q}$ estimates on the pressure and stopping time arguments.
\end{abstract}

Keywords: Primitive Equations, Mathematical Geophysics, Well-Posedness, Nonlinear Stochastic Partial Differential Equations, Stochastic Evolution Equations, Anisotropic Estimates, Pressure Estimates.

MSC2010: 35Q86, 60H15, 35Q35

\footnotetext{
${ }^{*}$ To appear in Nonlinearity.
} 


\section{Introduction}

The Primitive Equations (PEs) are widely considered to be a fundamental model in the study of large scale oceanic and atmospheric dynamics. These systems form the analytical core of the most advanced general circulation models for the atmosphere (AGCMs) or oceans (OGCMs) or the coupled oceanic-atmospheric system (GCMs). Moreover, beyond their considerable significance in applications, the PEs have generated much interest from the mathematics community due to their rich nonlinear, nonlocal character and their anisotropic structure.

The physical derivation of the Primitive Equations goes back to the early 20th century [Bje04, Ric07]. It is based on a scale analysis that accounts for the relatively constant density of the ocean (in the atmosphere the density follows a linear profile) and the contrast between the vertical and horizontal scales (on the order of several kilometers vs. thousands of kilometers). We refer the reader to e.g. [Ped82] (or [Ric07]) for further physical background for the (deterministic) PEs.

Hence the Primitive Equations express very fundamental laws of physics and one may wonder what the motivations are for introducing uncertainty in "exact" model equations. The introduction of stochastic processes in weather and climate prediction is aimed at accounting for a number of uncertainties and errors:

1. These "exact" models are numerically intractable; they cannot be fully solved with present super computers (and will not be for any foreseeable future). For this reason some sort of statistical averages are needed corresponding to the parameterization of the effects of the small unresolved scales. Indeed when the full equations are averaged it is common to introduce volumic stochastic forcing terms as a partial closure. This is reflected in a growing physics literature on 'stochastic parameterization'. See, for example, [Ros77, LL79, MT92, BSLP09, ZF10].

2. The physics of these "exact" models is in fact far from being fully understood. For the atmosphere the most dramatic example of physical uncertainty is due to the radiation properties of the air (clouds) which produce the source term called $F_{T}$ in the atmospheric analogue of the equation (1.2d) below, that is the energy balance equation. Nowadays these uncertainties due to the radiation properties of the clouds are considered to be the most severe source of uncertainty in weather and climate modeling. Note that while our presentation focuses on the equations of the ocean (which are mathematically slightly simpler than the corresponding atmospheric or coupled oceanic-atmospheric equations) our general results and methods in this work apply also to these systems. ${ }^{1}$

Further and related physical background in this connection is the subject of [GHTT].

With this backdrop in mind we study in this work a stochastic version of the primitive equations and establish the global existence and uniqueness of solutions for a nonlinear, multiplicative white noise. While the mathematical theory is now extensive in the deterministic case the stochastic primitive equations are only receiving attention very recently. We next review this mathematical background and then conclude the introduction by describing our results and outlining the analysis carried out below.

\section{Previous Mathematical Work}

To the best of our knowledge the mathematical study of the Primitive Equations started in the early 1990s with a series of works [LTW92b, LTW92a, LTW93] establishing the existence for all time of weak solutions of these equations. Subsequent articles improved these results and obtained existence and uniqueness of more

\footnotetext{
${ }^{1}$ Physical uncertainties on the boundary of the oceanic or coupled oceanic-atmospheric system are also present and are responsible for another source of stochasticity in these models. The boundary conditions (1.4) on $\Gamma_{i}$ (which is physically the sea surface-air interface) are a simplified version of the more realistic boundary conditions

$$
\alpha_{\mathbf{v}}\left(\mathbf{v}-\mathbf{v}_{*}\right)+\partial_{z} \mathbf{v}=g_{\mathbf{v}}, \quad \alpha_{T}\left(T-T_{*}\right)+\partial_{z} T=g_{T} .
$$

In the form (1.1) the boundary condition expresses two fundamental aspects of oceanic-atmospheric interaction namely the driving force of the wind (the term $g_{\mathbf{v}}$ ) and the heating or cooling of the air by the ocean (the term $g_{T}$ ). These functions $g_{\mathbf{v}}$ and $g_{T}$ are not well known and are estimated by modelers through very rough averages. Thus the uncertainties of these functions lead to stochastic PDEs with white noise on the boundary a subject which we intend to pursue in future work.
} 
regular (strong) solutions, very similar to the results available for the incompressible Navier-Stokes equations (see e.g. [GGMRB01, HTZ03, PTZ08]). Recently, taking advantage of the fact that the pressure is essentially two-dimensional in the PEs (unlike the Navier-Stokes equations) global results for the existence of strong solutions of the full three dimensional PEs were established in [CT07] and independently in [Kob06, Kob07]. In subsequent work, [KZ07], a different proof was developed which allows one to treat non-rectangular domains as well as different, physically realistic, boundary conditions. All of these works make essential use of the fact that the pressure terms appearing in the PEs can be shown to be essentially independent of the vertical variable $z$. In the present work we follow an approach closer to that of [KZ07] in that we estimate the pressure directly via earlier results for the evolution Stokes equation as in [SvW86]. In any case the deterministic mathematical theory for the Primitive equations has now reached an advanced stage and we refer the reader to the survey articles [PTZ08, RTT08] for further references and background.

Notwithstanding these extensive results in the deterministic case, the theory for the stochastic Primitive Equations remains underdeveloped. A two dimensional version of the PEs has been studied in a simplified form in [EPT07, GHZ08] and more recently in [GHT11a, GHT11b] in the greater generality of physically relevant boundary conditions and nonlinear multiplicative noise. While the full three dimensional system has been studied in [GH09] following the methods in [CT07], this work covers only the case of additive noise. In this case the PEs can be directly studied pathwise via a classical change of variables. In this and a companion work [DGHT11] devoted to the local existence of solutions we depart from [GH09] and develop different methods which allow us, in particular, to consider a nonlinear multiplicative forcing structure. The present work may therefore be seen as the continuation of [DGHT11] which takes us from the local to the global existence of solutions.

In contrast to the primitive equations, the theory of the related stochastic Navier-Stokes equations has undergone substantial developments dating back to the 1970's with the initial work [BT73]. In this literature, as for the wider literature on stochastic PDEs (see [DPZ92]) two principal notions of solutions have been developed. On the one hand Martingale (or probabilistically weak) solutions treat the stochastic elements in the problem as an unknown. In this case, one typically establishes compactness in the space of probability laws associated to solutions. See e.g. [Vio76, Cru89, CG94, FG95, MR04]. On the other hand Pathwise (or probabilistically strong) solutions consider the driving noise as being fixed in advance. See, for example, [DPZ96, BF00, Bre00, BP00, FR02, DPD03, MR05, Shi07, FR08, GHZ09]. In this and the companion work [DGHT11] we concentrate on this latter notion.

\section{The Governing Equations}

Having described the background and motivations for this article, we now outline its content in more detail. The stochastic version of the Primitive Equations that we study below takes the form:

$$
\begin{gathered}
\partial_{t} \mathbf{v}+(\mathbf{v} \cdot \nabla) \mathbf{v}+w \partial_{z} \mathbf{v}+\frac{1}{\rho_{0}} \nabla p+f \mathbf{k} \times \mathbf{v}-\mu_{\mathbf{v}} \Delta \mathbf{v}-\nu_{\mathbf{v}} \partial_{z z} \mathbf{v}=F_{\mathbf{v}}+\sigma_{\mathbf{v}}(\mathbf{v}, T, S) \dot{W}_{1}, \\
\partial_{z} p=-\rho g \\
\nabla \cdot \mathbf{v}+\partial_{z} w=0 \\
\partial_{t} T+(\mathbf{v} \cdot \nabla) T+w \partial_{z} T-\mu_{T} \Delta T-\nu_{T} \partial_{z z} T=F_{T}+\sigma_{T}(\mathbf{v}, T, S) \dot{W}_{2}, \\
\partial_{t} S+(\mathbf{v} \cdot \nabla) S+w \partial_{z} S-\mu_{S} \Delta S-\nu_{S} \partial_{z z} S=F_{S}+\sigma_{S}(\mathbf{v}, T, S) \dot{W}_{3}, \\
\rho=\rho_{0}\left(1+\beta_{T}\left(T-T_{r}\right)+\beta_{S}\left(S-S_{r}\right)\right) .
\end{gathered}
$$

Here, $U:=(\mathbf{v}, T, S)=(u, v, T, S), p, \rho$ represent the horizontal velocity, temperature, salinity, pressure and density of the fluid under consideration; $\mu_{\mathbf{v}}, \nu_{\mathbf{v}}, \mu_{T}, \nu_{T}, \mu_{S}, \nu_{S}$ are (possibly anisotropic) coefficients of the eddy and molecular viscosity and the heat and saline diffusivity respectively; $f$ is the Coriolis parameter appearing in the antisymmetric term in (1.2a) and accounts for the earth's rotation in the momentum equations. The third component of the velocity field, $w$ is a 'diagnostic variable' in that it is determined directly from $\mathbf{v}$, the components of the horizontal velocity field (see (2.1b), below). The evolution equations (1.2) occurs for $\left(x_{1}, x_{2}, z\right)$ ranging over a cylindrical domain $\mathcal{M}=\mathcal{M}_{0} \times(-h, 0) ; \mathcal{M}_{0}$ is an open bounded 
subset of $\mathbb{R}^{2}$ with smooth boundary $\partial \mathcal{M}_{0}$. Note that $\nabla=\left(\partial_{1}, \partial_{2}\right)$ where $\partial_{1}, \partial_{2}$ are the partial derivatives in the (horizontal) $x_{1}, x_{2}$ directions; $\Delta=\partial_{1}^{2}+\partial_{2}^{2}$ is the horizontal Laplace operator.

The stochastic terms are driven by independent Gaussian white noise processes $\dot{W}_{j}$ which are formally delta correlated in time. The stochastic terms may be written formally in the expansion

$$
\left(\begin{array}{c}
\sigma_{\mathbf{v}}(U) \dot{W}_{1}(t, x) \\
\sigma_{T}(U) \dot{W}_{2}(t, x) \\
\sigma_{S}(U) \dot{W}_{3}(t, x)
\end{array}\right)=\sum_{k \geq 1}\left(\begin{array}{c}
\sigma_{\mathbf{v}}^{k}(U)(x, t) \dot{W}_{1}^{k}(t) \\
\sigma_{T}^{k}(U)(x, t) \dot{W}_{2}^{k}(t) \\
\sigma_{S}^{k}(U)(x, t) \dot{W}_{3}^{k}(t)
\end{array}\right)
$$

where the elements $\dot{W}_{j}^{k}$ are independent white (in time) noise processes. We understand (1.2) in the Itō sense but the classical correspondence between the Itō and Stratonovich systems would allow one to treat both situations with the analysis herein. We recall the basic mathematical definitions and give precise conditions on the operators $\sigma_{\mathbf{v}}, \sigma_{T}, \sigma_{S}$ below.

The boundary $\partial \mathcal{M}$ is partitioned into the top $\Gamma_{i}=\mathcal{M}_{0} \times\{0\}$, the bottom $\Gamma_{b}=\mathcal{M}_{0} \times\{-h\}$ and the sides $\Gamma_{l}=\partial \mathcal{M}_{0} \times(-h, 0)$. We denote by $\mathbf{n}_{H}$ the outward unit normal to $\partial \mathcal{M}_{0}$. We prescribe the following boundary conditions:

$$
\partial_{z} \mathbf{v}=0, \quad w=0, \quad \partial_{z} T=0, \quad \partial_{z} S=0
$$

on $\Gamma_{i}$. At the bottom $\Gamma_{b}$ we take

$$
\partial_{z} \mathbf{v}=0, \quad w=0, \quad \partial_{z} T=0, \quad \partial_{z} S=0
$$

Finally for the lateral boundary $\Gamma_{l}$

$$
\mathbf{v}=0, \quad \partial_{\mathbf{n}_{H}} T=0, \quad \partial_{\mathbf{n}_{H}} S=0 .
$$

The equations and boundary conditions $(1.2),(1.4),(1.5),(1.6)$ are supplemented by initial conditions for $\mathbf{v}, T$ and $S$, that is

$$
\mathbf{v}=\mathbf{v}_{0}, \quad T=T_{0}, \quad S=S_{0}, \quad \text { at } t=0 .
$$

The Cauchy problem (1.2)-(1.7) given above models regional oceanic flows. We note however that equations of a quite similar structure may be given that describe the atmosphere and the coupled oceanic atmospheric system. See e.g. [PTZ08]. The methods developed here could thus be extended to treat these systems.

The manuscript is organized as follows. In the initial Section 2 we set the mathematical background for the work defining precisely the notion of pathwise solutions we are studying. Section 3 recalls the results in [DGHT11] that guarantee the local existence of solutions. Crucially, these results imply a maximal time of existence $\xi=\xi(\omega)$; on those samples $\omega$ (in the probability space $\Omega$ ) where $\xi(\omega)<\infty$ certain norms of the solution $U=(\mathbf{v}, T, S)$, in particular the $H^{1}$ norm, must blow up at $\xi(\omega)$. Having exhibited these preliminaries we next introduce a criteria for global existence based on the uniform control in time of $\mathbf{v} \in L^{4}$ and $\partial_{z} U \in L^{2}$. This sets the agenda for the remainder of the paper. In Section 4 we carry out the estimates in $L^{4}$. To this end we introduce a new 'shifted' variable $\hat{\mathbf{v}}$ that satisfies a random PDE that we may analyze pathwise. In this way we are able to handle the pressure via the results in [SvW86]. On the other hand a number of new terms appear in the nonlinear portion of the equations for $\hat{\mathbf{v}}$ that we must tackle. In Section 5 we turn to the estimates for $\partial_{z} U$ in $L^{2}$. In this case the pressure disappears when we exhibit the evolution for $\left|\partial_{z} U\right|_{L^{2}}^{2}$. As such we carry out the estimates in the original variable using stochastic methods: Itō calculus, the Burkholder-Davis-Gundy inequality, etc. Finally we include in an appendix further details for various technical complements used in the body of the work: the pressure estimates for the Stokes equations after [SvW86] and a particular version of the Gronwall lemma that we use to close the $L^{4}$ estimates in Section 4. 


\section{Mathematical Background and Notational Conventions}

In order to introduce a precise mathematical definition of solutions for the stochastic Primitive Equations we begin by rewriting (1.2) in a slightly different form. This formulation will be the basis for all that follows below:

$$
\begin{gathered}
\partial_{t} \mathbf{v}+(\mathbf{v} \cdot \nabla) \mathbf{v}+w(\mathbf{v}) \partial_{z} \mathbf{v}+\frac{1}{\rho_{0}} \nabla p_{s}-g \int_{z}^{0}\left(\beta_{T} \nabla T+\beta_{S} \nabla S\right) d \bar{z} \\
+f \mathbf{k} \times \mathbf{v}-\mu_{\mathbf{v}} \Delta \mathbf{v}-\nu_{\mathbf{v}} \partial_{z z} \mathbf{v}=F_{\mathbf{v}}+\sigma_{\mathbf{v}}(\mathbf{v}, T, S) \dot{W}_{1} \\
w(\mathbf{v})=\int_{z}^{0} \nabla \cdot \mathbf{v} d \bar{z}, \quad \int_{-h}^{0} \nabla \cdot \mathbf{v} d \bar{z}=0 \\
\partial_{t} T+(\mathbf{v} \cdot \nabla) T+w(\mathbf{v}) \partial_{z} T-\mu_{T} \Delta T-\nu_{T} \partial_{z z} T=F_{T}+\sigma_{T}(\mathbf{v}, T, S) \dot{W}_{2}, \\
\partial_{t} S+(\mathbf{v} \cdot \nabla) S+w(\mathbf{v}) \partial_{z} S-\mu_{S} \Delta S-\nu_{S} \partial_{z z} S=F_{S}+\sigma_{S}(\mathbf{v}, T, S) \dot{W}_{3}
\end{gathered}
$$

Here, as above in $(1.2), U:=(\mathbf{v}, T, S)=(u, v, T, S)$, are the horizontal velocity, temperature and salinity of the fluid under consideration, $\mu_{\mathbf{v}}, \nu_{\mathbf{v}}, \mu_{T}, \nu_{T}, \mu_{S}, \nu_{S}$ are the coefficients of the eddy and molecular viscosity and the heat and saline diffusivity respectively, $f$ is the Coriolis (rotation) parameter. By integrating $(1.2 \mathrm{~b})$ and making use of the relation $(1.2 \mathrm{f})$ we find that the pressure $p$ may be decomposed into a 'surface pressure', $p_{s}$ and some lower order terms that couple the momentum equations to those for the temperature and salinity. Crucially, we note that $p_{s}$ does not depend on the vertical variable $z$. Of course, this system (2.1) is supplemented with initial and boundary conditions as given in (1.7) and (1.4)-(1.6) above.

\subsection{Abstract Setting for the Equations}

We next recall the abstract setting for (2.1) (equivalently (1.2)). Note that our presentation and notations closely follow the recent survey [PTZ08].

Let us first recall some Hilbert spaces associated to (2.1). Define

$H:=\left\{(\mathbf{v}, T, S) \in\left(L^{2}(\mathcal{M})\right)^{4}: \nabla \cdot \int_{-h}^{0} \mathbf{v} d z=0\right.$ in $\mathcal{M}_{0}, \mathbf{n}_{H} \cdot \int_{-h}^{0} \mathbf{v} d z=0$ on $\left.\partial \mathcal{M}_{0}, \int_{\mathcal{M}} T d \mathcal{M}=\int_{\mathcal{M}} S d \mathcal{M}=0\right\}$

We equip this space with the classical $L^{2}$ inner product ${ }^{2}$ which we denote by $|\cdot|$. Define $P_{H}$ to be the Leray type projection operator from $L^{2}(\mathcal{M})^{4}$ onto $H$. For $H^{1}(\mathcal{M})^{4}$ we consider the subspace:

$$
V:=\left\{(\mathbf{v}, T, S) \in\left(H^{1}(\mathcal{M})\right)^{4}: \nabla \cdot \int_{-h}^{0} \mathbf{v} d z=0 \text { in } \mathcal{M}_{0}, \mathbf{v}=0 \text { on } \Gamma_{l}, \int_{\mathcal{M}} T d \mathcal{M}=\int_{\mathcal{M}} S d \mathcal{M}=0\right\}
$$

We equip $V$ with the inner product

$$
\begin{aligned}
\left(\left(U, U^{\sharp}\right)\right) & :=\left(\left(\mathbf{v}, \mathbf{v}^{\sharp}\right)\right)_{1}+\left(\left(T, T^{\sharp}\right)\right)_{2}+\left(\left(S, S^{\sharp}\right)\right)_{3}, \\
((\mathbf{v}, \mathbf{v}))_{1} & :=\int_{\mathcal{M}}\left(\mu_{\mathbf{v}} \nabla \mathbf{v} \cdot \nabla \mathbf{v}^{\sharp}+\nu_{\mathbf{v}} \partial_{z} \mathbf{v} \cdot \partial_{z} \mathbf{v}^{\sharp}\right) d \mathcal{M}, \\
\left(\left(T, T^{\sharp}\right)\right)_{2} & :=\int_{\mathcal{M}}\left(\mu_{T} \nabla T \cdot \nabla T^{\sharp}+\nu_{T} \partial_{z} T \cdot \partial_{z} T^{\sharp}\right) d \mathcal{M}, \\
\left(\left(S, S^{\sharp}\right)\right)_{3} & :=\int_{\mathcal{M}}\left(\mu_{S} \nabla S \cdot \nabla S^{\sharp}+\nu_{S} \partial_{z} S \cdot \partial_{z} S^{\sharp}\right) d \mathcal{M},
\end{aligned}
$$

\footnotetext{
${ }^{2}$ One sometimes also finds the more general definition $\left(U, U^{\sharp}\right):=\int_{\mathcal{M}}\left(\mathbf{v} \cdot \mathbf{v}^{\sharp} d+\kappa_{T} T T^{\sharp}+\kappa_{S} S S^{\sharp}\right) d \mathcal{M}$ with $\kappa_{T}, \kappa_{S}>0$ fixed constants. These parameters $\kappa_{T}, \kappa_{S}$ are useful for the coherence of physical dimensions and for (mathematical) coercivity. Since this is not needed here we take $\kappa_{T}=\kappa_{S}=1$. Similar remarks also apply to the space $V$.
} 
and take $\|\cdot\|=\sqrt{((\cdot, \cdot))}$. Note that under these definitions a Poincaré type inequality $|U| \leq c\|U\|$ holds for all $U \in V$. We take $V_{(2)}$ to be the closure of $V \cap C^{\infty}(\overline{\mathcal{M}})^{4}$ in $\left(H^{2}(\mathcal{M})\right)^{4}$ and equip this space with the classical $H^{2}(\mathcal{M})$ norm and inner product.

In the course of the analysis below we shall make estimates involving the individual components of the solution $U=(u, v, T, S)$. As such we shall sometime abuse notation and use $|\cdot|$ and $\|\cdot\|$ in the obvious way for $\mathbf{v}=(u, v), T$ or $S$. We shall also work with the $L^{p}=L^{p}(\mathcal{M})$ norms of these individual components of the solution. For $p \geq 1$ we denote $\mathbf{v}^{p}=\left(u|u|^{p-1}, v|v|^{p-1}\right)$ and let $|\mathbf{v}|_{L^{p}}:=\left(\int_{\mathcal{M}}\left(|u|^{p}+|v|^{p}\right) d \mathcal{M}\right)^{1 / p}$. Furthermore, for $q, p \geq 1$, we write

$$
|\mathbf{v}|_{L_{\mathbf{x}}^{q} L_{z}^{p}}:=\left(\int_{\mathcal{M}_{0}}\left(\int_{-h}^{0}\left(|u|^{p}+|v|^{p}\right) d z\right)^{q / p} d \mathcal{M}_{0}\right)^{1 / q}
$$

Remark 2.1. Assume that $\mathbf{v} \in H^{1}(\mathcal{M})$, with $\mathbf{v}=0$ on $\Gamma_{l}$ or $\int_{\mathcal{M}} \mathbf{v} d \mathcal{M}=0$. As in [PTZ08], an elementary calculation that makes use of the Sobolev embedding theorem in $\mathbb{R}^{2}$, reveals that

$$
|\mathbf{v}|_{L_{\mathbf{x}}^{q} L_{z}^{2}} \leq c|\mathbf{v}|^{1-s}\|\mathbf{v}\|^{s}
$$

where $q \geq 2$ and $s=1-2 / q$. This observation will be used on several occasions below.

The principal linear portion of the equation is defined by ${ }^{3}$

$$
A U=P_{H}\left(\begin{array}{c}
-\mu_{\mathbf{v}} \Delta \mathbf{v}-\nu_{\mathbf{v}} \partial_{z z} \mathbf{v} \\
-\mu_{T} \Delta T-\nu_{T} \partial_{z z} T \\
-\mu_{S} \Delta S-\nu_{S} \partial_{z z} S
\end{array}\right), \quad \text { for any } U=(\mathbf{v}, T, S) \in D(A)
$$

where:

$$
\begin{aligned}
D(A)=\left\{U=(\mathbf{v}, T) \in V_{(2)}\right. & : \partial_{z} \mathbf{v}=\partial_{z} T=\partial_{z} S=0 \text { on } \Gamma_{i}, \\
& \left.\partial_{\mathbf{n}_{H}} T=\partial_{\mathbf{n}_{H}} S=0 \text { on } \Gamma_{l}, \partial_{z} \mathbf{v}=\partial_{z} T=\partial_{z} S=0 \text { on } \Gamma_{b}\right\} .
\end{aligned}
$$

We observe that $A$ is self adjoint, positive definite. Indeed, by integration by parts and using the boundary conditions imposed by $(2.3)$, we see that $\left\langle A U, U^{\sharp}\right\rangle=\left(\left(U, U^{\sharp}\right)\right)$ for all $U, U^{\sharp} \in D(A)$ and thus, by density, for all $U, U^{\sharp} \in V$. Note also that, due to regularity results for the Stokes problem of Geophysical Fluid Dynamics, $|A U| \cong|U|_{H^{2}}$. See [Zia95] and also [PTZ08].

We next turn to the quadratically nonlinear terms appearing in (2.1). Noting that there is no momentum equation for $w$ in (2.1) and in accordance with (2.1b) we define the diagnostic function:

$$
w(U)=w(\mathbf{v}):=\int_{z}^{0} \nabla \cdot \mathbf{v} d \bar{z}, \quad U=(\mathbf{v}, T, S) \in V .
$$

Take, for $U, U^{\sharp} \in D(A)$ :

$$
B_{1}\left(U, U^{\sharp}\right):=P_{H}\left(\begin{array}{c}
(\mathbf{v} \cdot \nabla) \mathbf{v}^{\sharp} \\
(\mathbf{v} \cdot \nabla) T^{\sharp} \\
(\mathbf{v} \cdot \nabla) S^{\sharp}
\end{array}\right), \quad B_{2}\left(U, U^{\sharp}\right):=P_{H}\left(\begin{array}{c}
w(\mathbf{v}) \partial_{z} \mathbf{v}^{\sharp} \\
w(\mathbf{v}) \partial_{z} T^{\sharp} \\
w(\mathbf{v}) \partial_{z} S^{\sharp}
\end{array}\right) .
$$

We let $B\left(U, U^{\sharp}\right):=B_{1}\left(U, U^{\sharp}\right)+B_{2}\left(U, U^{\sharp}\right)$, and often write $B(U)=B(U, U)$. As in [PTZ08] one may show that $B$ is well defined as an element in $H$ for any $U, U^{\sharp} \in D(A)$ or $V_{(2)}$. In addition to the properties of $B$ appearing in [PTZ08] we have the following additional bounds, which are established with anisotropic estimates along the same lines (cf. Remark 2.1).

\footnotetext{
${ }^{3}$ In comparison to previous works, such as [PTZ08], we do not include all of the terms due to the pressure in the definition of $A$. Such elements destroy the symmetry of $A$ and are therefore relegated to a lower order term $A_{p}$. See (2.7).
} 
Lemma 2.1. Suppose that $U, U^{\sharp} \in D(A)$ and that $U^{b} \in H$. Then

$$
\left|\left\langle B\left(U, U^{\sharp}\right), U^{\mathrm{b}}\right\rangle\right| \leq c\left(|\mathbf{v}|_{L^{4}}\left\|U^{\sharp}\right\|^{1 / 4}\left|A U^{\sharp}\right|^{3 / 4}\left|U^{\mathrm{b}}\right|+\|\mathbf{v}\|^{1 / 2}|\mathbf{v}|_{(2)}^{1 / 2}\left|\partial_{z} U^{\sharp}\right|^{1 / 2}\left\|\partial_{z} U^{\sharp}\right\|^{1 / 2}\left|U^{\mathrm{b}}\right|\right) .
$$

For the second component of the pressure in (2.1a) we take

$$
A_{p} U=P_{H}\left(\begin{array}{c}
-g \int_{z}^{0}\left(\beta_{T} \nabla T+\beta_{S} \nabla S\right) d \bar{z} \\
0 \\
0
\end{array}\right), \quad U \in V .
$$

We capture the Coriolis (rotational) forcing according to

$$
E U=P_{H}\left(\begin{array}{c}
f \mathbf{k} \times v \\
0 \\
0
\end{array}\right), \quad U \in H .
$$

Finally we set

$$
F=P_{H}\left(\begin{array}{c}
F_{\mathbf{v}} \\
F_{T} \\
F_{S}
\end{array}\right)
$$

We shall assume throughout this work that:

$$
F \in L^{2}\left(\Omega ; L_{l o c}^{2}\left([0, \infty) ; L^{4}(\mathcal{M})\right)\right) .
$$

We finally give a precise definition for the stochastic terms appearing in (2.1) (i.e. (1.2)). For this purpose let us briefly recall some aspects of the theory of the infinite dimensional Itō integration. As we are studying pathwise solutions of (1.2) (see Definitions 2.1, 2.2 below) we shall fix throughout this work a single stochastic basis $\mathcal{S}:=\left(\Omega, \mathcal{F}, \mathbb{P},\left\{\mathcal{F}_{t}\right\}_{t \geq 0}, W\right)$. Here $W$ is a cylindrical brownian motion defined on an auxiliary Hilbert space $\mathfrak{U}$ and adapted to the filtration $\left\{\mathcal{F}_{t}\right\}_{t \geq 0}$. By picking a complete orthonormal basis $\left\{e_{k}\right\}_{k \geq 1}$ for $\mathfrak{U}, W$ may be written as the formal sum $W(t, \omega)=\sum_{k \geq 1} e_{k} W_{k}(t, \omega)$ where the elements $W_{k}$ are an independent sequence of $1 \mathrm{D}$ standard Brownian motions.

Consider another separable Hilbert space $X$ and let $L_{2}(\mathfrak{U}, X)=\left\{R \in \mathcal{L}(\mathfrak{U}, X): \sum_{k}\left|R e_{k}\right|^{2}<\infty\right\}$, that is the collection of Hilbert-Schmidt operators from $\mathfrak{U}$ into $X$. Given an $X$-valued predictable ${ }^{4}$ process $G \in L^{2}\left(\Omega ; L_{l o c}^{2}\left([0, \infty), L_{2}(\mathfrak{U}, X)\right)\right)$ one may define the (Itō) stochastic integral

$$
M_{t}:=\int_{0}^{t} G d W=\sum_{k} \int_{0}^{t} G_{k} d W_{k}
$$

as an element in $\mathcal{M}_{X}^{2}$, that is the space of all $X$-valued square integrable martingales (see [DPZ92] or [PR07]); here $G_{k}=G e_{k}$. The process $\left\{M_{t}\right\}_{t \geq 0}$ has many desirable properties. Most notably for the analysis here, the Burkholder-Davis-Gundy inequality holds which in the present context takes the form,

$$
\mathbb{E}\left(\sup _{t \in[0, T]}\left|\int_{0}^{t} G d W\right|_{X}^{r}\right) \leq c \mathbb{E}\left(\int_{0}^{T}|G|_{L_{2}(\mathfrak{U}, X)}^{2} d t\right)^{r / 2},
$$

valid for any $r \geq 1$. Here $c$ is an absolute constant depending only on $r$.

\footnotetext{
${ }^{4}$ Let $\Phi=\Omega \times[0, \infty)$ and take $\mathcal{G}$ to be the $\sigma$-algebra generated by sets of the form$$
(s, t] \times F, \quad 0 \leq s<t<\infty, F \in \mathcal{F}_{s} ; \quad\{0\} \times F, \quad F \in \mathcal{F}_{0} .
$$

Recall that an $X$ valued process $U$ is called predictable (with respect to the stochastic basis $\mathcal{S}$ ) if it is measurable from $(\Phi, \mathcal{G}$ ) into $(X, \mathcal{B}(X)), \mathcal{B}(X)$ being the family of Borel sets of $X$.
} 
Given any pair of Banach spaces $\mathcal{X}$ and $\mathcal{Y}$ we denote by $\operatorname{Bnd}_{u}(\mathcal{X}, \mathcal{Y})$, the collection of all continuous mappings $\Psi:[0, \infty) \times \mathcal{X} \rightarrow \mathcal{Y}$ such that

$$
\|\Psi(x, t)\|_{\mathcal{Y}} \leq c\left(1+\|x\|_{\mathcal{X}}\right), \quad x \in \mathcal{X}, t \geq 0
$$

where the numerical constant $c$ may be chosen independently of $t$. If, in addition,

$$
\|\Psi(x, t)-\Psi(y, t)\|_{\mathcal{Y}} \leq c\|x-y\|_{\mathcal{X}}, \quad x, y \in \mathcal{X}, t \geq 0,
$$

we say $\Psi$ is in $\operatorname{Lip}_{u}(\mathcal{X}, \mathcal{Y})$. We define

$$
\sigma((\mathbf{v}, T, S))=\sigma(U)=P_{H}\left(\begin{array}{c}
\sigma_{\mathbf{v}}(U) \\
\sigma_{T}(U) \\
\sigma_{S}(U)
\end{array}\right), \quad U \in H,
$$

and assume that $\sigma:[0, \infty) \times H \rightarrow L_{2}(\mathfrak{U}, H)$ with

$$
\sigma \in \operatorname{Lip}_{u}\left(H, L_{2}(\mathfrak{U}, H)\right) \cap \operatorname{Lip}_{u}\left(V, L_{2}(\mathfrak{U}, V)\right) \cap \operatorname{Bnd}_{u}\left(V, L_{2}(\mathfrak{U}, D(A))\right) .
$$

Under the assumption (2.13) on $\sigma$, the stochastic integral $t \mapsto \int_{0}^{t} \sigma(U) d W$ may be shown to be well defined (in the Itō sense), taking values in $H$ whenever $U \in L^{2}\left(\Omega, L_{l o c}^{2}([0, \infty) ; H)\right)$ and is predictable.

Remark 2.2. The condition (2.13) may be shown to cover a wide class of examples, including but not limited to the classical cases of additive and linear multiplicative noise, projections of the solution in any direction, and directional forcings of Lipschitz functionals of the solution. See [GHTT] for further details and physical motivations.

We note that the final condition $\sigma \in \operatorname{Bnd}_{u}\left(V, L_{2}(\mathfrak{U}, D(A))\right)$ in fact may be weakened slightly to $\sigma \in$ $\operatorname{Bnd}_{u}\left(D(A), L_{2}(\mathfrak{U}, D(A))\right)$ for the proof of local existence. However for the global existence we need this stronger condition in order to carry out the $L^{4}$ estimates which appear in Section 4. In fact the stronger condition used in this section is an artifact of a 'pathwise' approach which further complicates the nonlinear term. This pathwise approach is necessitated by the appearance of certain terms explicitly involving the pressure $p_{s}$.

Collecting the operators defined above we reformulate (2.1) (equivalently, (1.2)) as the following abstract evolution system

$$
d U+\left(A U+B(U)+A_{p} U+E U\right) d t=F d t+\sigma(U) d W, \quad U(0)=U_{0} .
$$

We recall the following pathwise (or probabilistically strong) notions of local and global existence for this system.

Definition 2.1 (Local Solutions). Let $\mathcal{S}=\left(\Omega, \mathcal{F},\left\{\mathcal{F}_{t}\right\}_{t>0}, \mathbb{P}, W\right)$ be a fixed stochastic basis and suppose that $U_{0}$ is a $V$ valued, $\mathcal{F}_{0}$ measurable random variable with $\mathbb{E}\left\|U_{0}\right\|^{2}<\infty$. Assume that $F$ satisfies (2.10) and that (2.13) holds for $\sigma$.

(i) A pair $(U, \tau)$ is a a local strong pathwise solution of (2.14) (i.e. of (1.2)) if $\tau$ is a strictly positive stopping time and $U(\cdot \wedge \tau)$ is an $\mathcal{F}_{t}$-adapted process in $V$ so that (relative to the fixed basis $\mathcal{S}$ )

$$
U(\cdot \wedge \tau) \in L^{2}(\Omega ; C([0, \infty) ; V)), \quad U \mathbb{1}_{t \leq \tau} \in L^{2}\left(\Omega ; L_{l o c}^{2}([0, \infty) ; D(A))\right) ;
$$

and, for every $t \geq 0$,

$$
U(t \wedge \tau)+\int_{0}^{t \wedge \tau}\left(A U+B(U)+A_{p} U+E U\right) d t^{\prime}=U_{0}+\int_{0}^{t \wedge \tau} F d t^{\prime}+\int_{0}^{t \wedge \tau} \sigma(U) d W,
$$

with equality understood in $H$. 
(ii) Strong pathwise solutions of (2.14) are said to be (pathwise) unique up to a stopping time $\tau>0$ if given any pair of solutions $\left(U^{1}, \tau\right),\left(U^{2}, \tau\right)$ which coincide at $t=0$ on the event $\tilde{\Omega}=\left\{U^{1}(0)=U^{2}(0)\right\} \subseteq \Omega$, then

$$
\mathbb{P}\left(\mathbb{1}_{\tilde{\Omega}}\left(U^{1}(t \wedge \tau)-U^{2}(t \wedge \tau)\right)=0 ; \forall t \geq 0\right)=1 .
$$

Remark 2.3. For a local solution $(U, \tau)$ consider the increasing function

$$
\phi(\sigma):=\sup _{t \in[0, \sigma]}\|U(t)\|^{2} \quad(\text { defined for } \sigma \in[0, \tau]) .
$$

We infer from (2.15) that $\phi(\tau)<\infty$, almost surely. Of course the question arrises as to whether the solution $U$ and therefore $\phi$ can be extended beyond $\tau$. We will call $\xi$ the supremum of the $\tau$ 's such that $(U, \tau)$ is a local solution of (2.14). A priori, $\xi$ might be finite or infinite and we are lead to the following notions of maximal and global solutions.

Definition 2.2 (Maximal and Global Solutions).

(i) Suppose that $\left\{\tau_{n}\right\}_{n \geq 1}$ is a an increasing sequence of stopping times converging to a (possibly infinite) stopping time $\xi$ and assume that $U$ is a predictable process in $H$. We say that the pair $(U, \xi)$ is a maximal pathwise strong solution if $\left(U, \tau_{n}\right)$ is a local strong pathwise solution for each $n$ and

$$
\phi(\xi)=\sup _{t \in[0, \xi]}\|U\|^{2}=\infty
$$

almost surely on the set $\{\xi<\infty\}$.

(ii) If $(U, \xi)$ is a maximal pathwise strong solution and $\xi=\infty$ a.s. then we say that the solution is global. Note that $\phi(\infty)$ can be finite or infinite in this case.

With these foundations in place we finally state, in precise terms, the main result of the work, the global well-posedness of (1.2) in the class of pathwise, strong solutions:

Theorem 2.1. Suppose that $U_{0} \in L^{2}(\Omega, V)$ and is $\mathcal{F}_{0}$ measurable. Assume that $F$ satisfies (2.10) and that (2.13) holds for $\sigma$. Then, there exists a unique global pathwise solution $U$ of $(2.14)$ with $U(0)=U_{0}$.

The proof of this Theorem is given below at the end of Section 3. It follows directly from the combination of Theorems 3.1, 3.2 and Propositions 4.1, 5.1.

\section{Local Existence and A Criteria For Global Existence}

As with the deterministic case, the first step in the analysis is to establish the local existence of solutions all the way to a 'maximal' existence time $\xi>0$. Recall that if $\xi(\omega)<\infty$ for $\omega \in \Omega$, then the $H^{1}$ norm of the solution must blow up at this maximal time as expressed by (2.17). With this in hand, we next show in Theorem 3.2 that the strong norms of solutions may be controlled by a combination of the $L^{4}$ norm of the momentum $\mathbf{v}$ and by the vertical gradient of the entire solution $U$. The results in Theorem 3.2 establish a sufficient condition for Theorem 2.1 to be valid and thus set the program for the rest of the article.

The first step, establishing the existence of a maximal pathwise solution, is given by Theorem 3.1 immediately below. The proof of this result follows immediately from abstract results in [DGHT11, Theorem 2.1 and Section 6].

Theorem 3.1. Suppose that (2.10) and (2.13) hold for $F$ and $\sigma$ respectively. Given any $\mathcal{F}_{0}$-measurable $U_{0} \in L^{2}(\Omega, V)$, there exists a unique maximal pathwise solution $(U, \xi)$ of $(2.14)$ with $U(0)=U_{0}$. If, for some $p \geq 2, U(0) \in L^{p}(\Omega, V)$ then, for any $t>0$, we have the bounds

$$
\mathbb{E}\left(\sup _{t^{\prime} \in[0, t \wedge \xi]}|U|^{p}+\int_{0}^{t \wedge \xi}\|U\|^{2}|U|^{p-2} d t^{\prime}\right)<\infty,
$$


and

$$
\mathbb{E}\left(\int_{0}^{t \wedge \xi}\|U\|^{2} d t^{\prime}\right)^{p / 2}<\infty .
$$

Remark 3.1. The local existence of solutions for a (nonlinear) multiplicative noise is more challenging than the additive case. In this latter situation where the noise terms have no state dependence, a simple change of variables which 'subtracts off' the noise can be used to transform the governing equations into a random PDE which can be treated using the methods of ordinary calculus. Here, crucially, the probabilistic dependence, given as $\omega \in \Omega$, may be essentially treated as a parameter in the problem.

In the general case of nonlinear multiplicative noise no such transformation is available. As a consequence stochastic integral terms must be estimated directly and as such the classical compactness methods break down since the problem may no longer be handled with the 'pathwise' approach typically used in the additive case. More involved probabilistic machinery is therefore needed. The proof of Theorem 3.1 appearing in [DGHT11] is based on an elementary but powerful characterization of convergence in probability given in [GK96]. With this characterization we were able to establish a Yamada-Watanabe type result namely: 'pathwise' solutions follow from the existence of Martingale solutions when we have pathwise uniqueness for solutions (see Definition ??,(ii)). A different method based on the Cauchy convergence of the Galerkin solutions associated to the basic SPDE is developed in [GHZ09] for the Navier-Stokes equations in dimensions D=2,3 and for the $2 D$ Primitive equations in [GHT11a, GHT11b].

For any give $K>0$ we define the following stopping times which are used here and below:

$$
\begin{aligned}
\tau_{K}^{W} & :=\inf _{t \geq 0}\left\{\sup _{t^{\prime} \in[0, t \wedge \xi]}|U|^{2}+\int_{0}^{t \wedge \xi}\left(\|U\|^{2}+|F|_{L^{4}}^{2}\right) d t^{\prime} \geq K\right\} \\
& =\sup _{t \geq 0}\left\{\sup _{t^{\prime} \in[0, t \wedge \xi]}|U|^{2}+\int_{0}^{t \wedge \xi}\left(\|U\|^{2}+|F|_{L^{4}}^{2}\right) d t^{\prime} \leq K\right\}
\end{aligned}
$$

and also

$$
\begin{aligned}
& \tau_{K}^{(1)}:=\inf _{t \geq 0}\left\{\sup _{t^{\prime} \in[0, t \wedge \xi]}|\mathbf{v}|_{L^{4}}^{4} \geq K\right\}=\sup _{t \geq 0}\left\{\sup _{t^{\prime} \in[0, t \wedge \xi]}|\mathbf{v}|_{L^{4}}^{4} \leq K\right\}, \\
& \tau_{K}^{(2)}:=\inf _{t \geq 0}\left\{\sup _{t^{\prime} \in[0, t \wedge \xi]}\left|\partial_{z} U\right|^{2}+\int_{0}^{t \wedge \xi}\left\|\partial_{z} U\right\|^{2} \geq K\right\}=\sup _{t \geq 0}\left\{\sup _{t^{\prime} \in[0, t \wedge \xi]}\left|\partial_{z} U\right|^{2}+\int_{0}^{t \wedge \xi}\left\|\partial_{z} U\right\|^{2} \leq K\right\}, \\
& \tau_{K}:=\tau_{K}^{(1)} \wedge \tau_{K}^{(2)}
\end{aligned}
$$

Note here that we employ notation typically used in optimization (see e.g. [ET99, Roc70, Pol71, Pol97]) and we follow the convention that the infimum of an empty set is $+\infty$. By Theorem 3.1 and the standing assumption (2.10), it is direct to infer that $\lim _{K \uparrow \infty} \tau_{K}^{W}=\infty$. We establish below, by gathering together the conclusions in Propositions 4.1, 5.1, that $\lim _{K \uparrow \infty} \tau_{K}=\infty$. This, as the next result shows, is a sufficient condition for the global existence of solutions of (1.2).

We observe and emphasize that the definition of $\tau_{K}$ does not imply that $\tau_{K} \leq \xi$ a.s. Indeed, it could be possible that $\tau_{K}=\infty$ if the sets defining $\tau_{K}$ are empty. Rather, that $\tau_{K} \leq \xi$, will be proven as part of the next theorem.

Theorem 3.2. The hypotheses are the same as in Theorem 3.1 and we consider the maximal strong pathwise solution $(U, \xi)=((\mathbf{v}, T, S), \xi)$ of (1.2) define in this theorem. For any deterministic constant $K$, consider the stopping times $\tau_{K}$ defined according to (3.4). Then, for any deterministic time $t>0$ and any $K>0$,

$$
\mathbb{E}\left(\sup _{t^{\prime} \in\left[0, t \wedge \tau_{K} \wedge \xi\right]}\|U\|^{2}+\int_{0}^{t \wedge \tau_{K} \wedge \xi}|A U|^{2} d t^{\prime}\right)<\infty .
$$


Furthermore $\tau_{K} \leq \xi$ almost surely and consequently if we show (by other means) that $\lim _{K \uparrow} \tau_{K}=+\infty$ then $\xi=\infty$ a.s., that is they solution $U$ is global in the sense of Definition 2.2, (ii).

Proof. With an application of the Itō formula we deduce the following evolution equation for $\|U\|^{2}$ :

$$
d\|U\|^{2}+2|A U|^{2} d t=-2\left\langle B(U)+A_{p} U+E U+F, A U\right\rangle d t+\|\sigma(U)\|_{L_{2}(\mathfrak{U}, V)}^{2} d t+2\left\langle A^{1 / 2} \sigma(U), A^{1 / 2} U\right\rangle d W
$$

Fix any $0 \leq \tau_{a} \leq \tau_{b} \leq \tau_{K} \wedge \xi \wedge t$. Integrating in time, taking a supremum over the interval $\left[\tau_{a}, \tau_{b}\right]$ and then the expected value of the resulting expression yields the estimate

$$
\begin{aligned}
& \mathbb{E}\left(\sup _{\tau_{a} \leq t^{\prime} \leq \tau_{b}}\|U\|^{2}+2 \int_{\tau_{a}}^{\tau_{b}}|A U|^{2} d t^{\prime}\right) \\
& \leq \mathbb{E}\left\|U\left(\tau_{a}\right)\right\|^{2}+c \mathbb{E} \int_{\tau_{a}}^{\tau_{b}}|\langle B(U), A U\rangle| d t+c \mathbb{E} \int_{\tau_{a}}^{\tau_{b}}\left(\left|\left\langle A_{p} U+E U+F, A U\right\rangle\right|+\|\sigma(U)\|_{L_{2}(\mathfrak{U}, V)}^{2}\right) d t \\
& \quad+c \mathbb{E} \sup _{\tau_{a} \leq t^{\prime} \leq \tau_{b}}\left|\int_{\tau_{a}}^{t^{\prime}}\left\langle A^{1 / 2} \sigma(U), A^{1 / 2} U\right\rangle d W\right| \\
& :=\mathbb{E}\left\|U\left(\tau_{a}\right)\right\|^{2}+J_{1}+J_{2}+J_{3} .
\end{aligned}
$$

We address the terms $J_{k}$ in reverse order. For $J_{3}$ using the Burkholder-Davis-Gundy inequality, (2.11), and the assumption in $(2.13)$ that $\sigma \in \operatorname{Lip}_{u}\left(V, L_{2}(\mathfrak{U}, V)\right)$ we infer

$$
\begin{aligned}
J_{3} & \leq c \mathbb{E}\left(\int_{\tau_{a}}^{\tau_{b}}\left\langle A^{1 / 2} \sigma(U), A^{1 / 2} U\right\rangle^{2} d t\right)^{1 / 2} \leq c \mathbb{E}\left(\int_{\tau_{a}}^{\tau_{b}}\|\sigma(U)\|_{L_{2}(\mathfrak{U}, V)}^{2}\|U\|^{2} d t\right)^{1 / 2} \\
& \leq \frac{1}{2} \mathbb{E} \sup _{t^{\prime} \in\left[\tau_{a}, \tau_{b}\right]}\|U\|^{2}+c \mathbb{E} \int_{\tau_{a}}^{\tau_{b}}\left(1+\|U\|^{2}\right) d t .
\end{aligned}
$$

For $J_{2}$ we use again that $\sigma \in \operatorname{Lip}_{u}\left(V, L_{2}(\mathfrak{U}, V)\right)$ and make direct estimates for $A_{p}$ and $E$; see respectively, (2.7), (2.8) above. We infer

$$
\left|\left\langle A_{p} U+E U+F, A U\right\rangle\right|+\|\sigma(U)\|_{L_{2}(\mathfrak{U}, V)}^{2} \leq \frac{1}{2}|A U|^{2}+c\left(1+\|U\|^{2}+|F|^{2}\right) .
$$

On the other hand, (2.6) implies that

$$
\begin{aligned}
|\langle B(U), A U\rangle| & \left.\leq|\mathbf{v}|_{L^{4}}\|U\|^{1 / 4}|A U|^{7 / 4}+\|U\|^{1 / 2}\left|\partial_{z} U\right|^{1 / 2}\left\|\partial_{z} U\right\|^{1 / 2}|A U|^{3 / 2}\right) \\
& \leq \frac{1}{2}|A U|^{2}+c\left(|\mathbf{v}|_{L^{4}}^{8}+\left|\partial_{z} U\right|^{2}\left\|\partial_{z} U\right\|^{2}\right)\|U\|^{2} .
\end{aligned}
$$

This takes care of $J_{1}$. Combining these three estimates, we conclude that

$$
\begin{aligned}
& \mathbb{E}\left(\sup _{\tau_{a} \leq t^{\prime} \leq \tau_{b}}\|U\|^{2}+\int_{\tau_{a}}^{\tau_{b}}|A U|^{2} d t^{\prime}\right) \\
& \quad \leq c \mathbb{E}\left(\left\|U\left(\tau_{a}\right)\right\|^{2}+\int_{\tau_{a}}^{\tau_{b}}\left(|\mathbf{v}|_{L^{4}}^{8}+\left|\partial_{z} U\right|^{2}\left\|\partial_{z} U\right\|^{2}+1\right)\|U\|^{2} d t+\int_{\tau_{a}}^{\tau_{b}}|F|^{2} d t\right) .
\end{aligned}
$$

We now apply a stochastic version of the Gronwall lemma to (3.5) from [GHZ09, Lemma 5.3] which is recalled below in the Appendix as Proposition 6.3. Note carefully that the constants appearing in the above equation do not depend on $\tau_{a}, \tau_{b}$. By taking $X:=\|U\|^{2}, Y:=|A U|^{2}, Z:=|F|^{2}, R:=|\mathbf{v}|_{L^{4}}^{8}+\left|\partial_{z} U\right|^{2}\left\|\partial_{z} U\right\|^{2}+1$ and finally $\tau:=t \wedge \tau_{K} \wedge \xi$ in Proposition 6.3 we therefore derive (3.5) from (3.6).

We now argue by contradiction to show that $\tau_{K} \leq \xi$. If, on a set of non-zero measure, $\tau_{K}>\xi$ then using that $\left\{\tau_{K}>\xi\right\}=\cup_{t \geq 0}\left\{\tau_{K} \wedge t>\xi\right\}$ we would infer the existence of a deterministic time $t>0$ such that 
$\left\{\tau_{K} \wedge t>\xi\right\}$ is of non-zero measure. By the definition of $\xi$ (cf. (2.17)), we would therefore infer that for this $t>0$

$$
\sup _{t^{\prime} \in\left[0, t \wedge \tau_{K} \wedge \xi\right]}\|U\|^{2}+\int_{0}^{t \wedge \tau_{K} \wedge \xi}|A U|^{2} d t^{\prime} \geq \sup _{t^{\prime} \in[0, \xi]}\|U\|^{2}=\infty
$$

over $\left\{\tau_{K} \wedge t>\xi\right\}$. Since $\left\{\tau_{K} \wedge t>\xi\right\}$ is a set of non-zero measure we have a contradiction with (3.5). With this, the proof of Theorem 3.2 is therefore complete.

We conclude this section by explaining in precise terms how we will use Theorem 3.2 to prove Theorem 2.1 :

Proof of Theorem 2.1. Define the stopping times $\tau_{K}^{(1)}, \tau_{K}^{(2)}$, and take $\tau_{K}:=\tau_{K}^{(1)} \wedge \tau_{K}^{(2)}$ as in (3.4). According to Theorem 3.2, the desired result will follow once it is shown that $\lim _{K \uparrow \infty} \tau_{K}=\infty$ a.s. In Proposition 4.1 it is shown that $\lim _{K \uparrow \infty} \tau_{K}^{(1)}=\infty$ and from Proposition 5.1 we infer that $\lim _{K \uparrow \infty} \tau_{K}^{(2)}=\infty$. The proof is therefore complete.

\section{Estimates in $L^{4}(\mathcal{M})$}

In this section we establish that $\lim _{K \uparrow \infty} \tau_{K}^{(1)}=+\infty$ where $\tau_{K}^{(1)}$ is defined as in (3.4) above. This amounts to showing that the horizontal component of the velocity field, $\mathbf{v}$, of the solution $U=(\mathbf{v}, T, S)$ is almost surely finite in $L_{t}^{\infty} L_{\mathbf{x}, z}^{4}$, at least up to the maximal time of existence, $\xi$.

More precisely we will prove the following:

Proposition 4.1. Suppose that the conditions given in Theorem 2.1 hold and consider the resulting maximal strong solution $(U, \xi)=((\mathbf{v}, T, S), \xi)$ of $(1.2)$. Then, for any $t>0$,

$$
\sup _{t^{\prime} \in[0, t \wedge \xi]}\left|\mathbf{v}\left(t^{\prime}\right)\right|_{L^{4}}<\infty \quad \text { a.s. }
$$

Moreover, defining, for $K>0$ the stopping time $\tau_{K}^{(1)}$ as in (3.4) we have, up to a set of measure zero, that $\lim _{K \uparrow \infty} \tau_{K}^{(1)}=\infty$.

Proposition 4.1 will be an easy consequence of Propositions 4.2, 4.3 below. The proof relies on a decomposition of the solution $U$ of (2.16) into a sum of two components: $\check{U}=(\check{\mathbf{v}}, \check{T}, \check{S})$ and $\hat{U}=(\hat{\mathbf{v}}, \hat{T}, \hat{S}) ; \check{U}$ solves a linear stochastic system, (4.2), whereas $\hat{U}$ is the solution of the random PDE, (4.4). The later system may be studied 'pathwise', that is pointwise in $\Omega$. We are therefore able to treat certain terms involving the pressure explicitly by means of Proposition 6.1 (cf. [KZ07], [SvW86]). In any case, we are able to establish control on the norms $L_{\mathbf{x}, z}^{4}$ of $\check{\mathbf{v}}$ and $\hat{\mathbf{v}}$ as in (4.1) in Propositions 4.2, 4.3 from which Proposition 4.1 then follows immediately.

\section{The Decomposition}

We describe next the decomposition and recall some basic properties of the linear portion of this splitting. Consider the following linear system, an infinite dimensional Ornstein-Uhlenbeck process:

$$
d \check{U}+A \check{U} d t=\mathbb{1}_{t \leq \xi} \sigma(U) d W, \quad \check{U}(0)=0 .
$$

We underline here that the element $(U, \xi)$ appearing in the right hand side is the unique maximal solution of (1.2) corresponding to the given initial condition $U_{0}$.

Proposition 4.2. There exists a unique global, pathwise strong solution $\check{U}=(\check{\mathbf{v}}, \check{T}, \check{S})$ of $(4.2)$ with $\check{U}$ $\in L^{2}(\Omega ; C([0, \infty) ; D(A)))$. In particular, for any $t>0$,

$$
\sup _{t^{\prime} \in[0, t \wedge \xi]}\left|\check{\mathbf{v}}\left(t^{\prime}\right)\right|_{L^{4}}<\infty \quad \text { a.s. }
$$


This result is proven exactly as in [GHT11b, Lemma 4.1]. See also [DPZ92] for further generalities concerning infinite dimensional linear stochastic systems of the form (4.2).

Remark 4.1. The final condition in (2.13), $\sigma \in B n d\left(V, L_{2}(\mathfrak{U}, D(A))\right)$ is imposed so that $\check{U}$ is guaranteed to evolve continuously in $D(A)$. This stronger conditions is needed in several estimates below (see e.g. (4.11), (4.15)).

We next define $\hat{U}=U-\check{U}$. By subtracting (4.2) from (2.14) we find that $\hat{U}$ satisfies

$$
\begin{gathered}
\frac{d}{d t} \hat{U}+A \hat{U}+B(\hat{U}+\check{U})+E(\hat{U}+\check{U})+A_{p}(\hat{U}+\check{U})=F, \\
\hat{U}(0)=U(0)=U_{0} .
\end{gathered}
$$

The equation for the first component $\hat{\mathbf{v}}$ of the solution $\hat{U}=(\hat{\mathbf{v}}, \hat{T}, \hat{S})$ is given by

$$
\begin{aligned}
\partial_{t} \hat{\mathbf{v}}+((\hat{\mathbf{v}}+\check{\mathbf{v}}) \cdot \nabla)(\hat{\mathbf{v}}+\check{\mathbf{v}}) & +w(\hat{\mathbf{v}}+\check{\mathbf{v}}) \partial_{z}(\hat{\mathbf{v}}+\check{\mathbf{v}})+\frac{1}{\rho_{0}} \nabla \hat{p}_{s}+f \mathbf{k} \times(\hat{\mathbf{v}}+\check{\mathbf{v}})-\mu_{\mathbf{v}} \Delta \hat{\mathbf{v}}-\nu_{\mathbf{v}} \partial_{z z} \hat{\mathbf{v}} \\
& =F_{\mathbf{v}}+g \int_{z}^{0}\left(\beta_{T} \nabla(\hat{T}+\check{T})+\beta_{S} \nabla(\hat{S}+\check{S})\right) d \bar{z}
\end{aligned}
$$

Note that the diagnostic function, $w(\cdot)$ is defined as above in (2.4). As, with $\mathbf{v}$, we have $\int_{-h}^{0} \nabla \cdot \hat{\mathbf{v}} d \bar{z}=0$. Of course, (4.5) is supplemented with boundary conditions as in (1.4)-(1.6). We will hereafter prove:

Proposition 4.3. Define $(\hat{\mathbf{v}}, \hat{T}, \hat{S})=\hat{U}:=U-\check{U}$. Then for any, for any $t>0, \hat{\mathbf{v}}$ satisfies

$$
\sup _{t^{\prime} \in[0, t \wedge \xi]}\left|\hat{\mathbf{v}}\left(t^{\prime}\right)\right|_{L^{4}}<\infty \quad \text { a.s. }
$$

Proof. Note that (4.5) does not have any white noise terms. Multiplying (4.5) by $\hat{\mathbf{v}}^{3}=\left(v_{1}^{3}, v_{2}^{3}\right)$ and then integrating over the domain $\mathcal{M}$ leads to the following system describing the time evolution of $|\hat{\mathbf{v}}|_{L^{4}}^{4}$ :

$$
\begin{aligned}
\frac{1}{4} \frac{d}{d t}|\hat{\mathbf{v}}|_{L^{4}}^{4} & +3 \mu_{\mathbf{v}} \sum_{j, k} \int_{\mathcal{M}}\left(\partial_{j} \hat{v}_{k}\right)^{2} \hat{v}_{k}^{2} d \mathcal{M}+3 \nu_{\mathbf{v}} \sum_{k} \int_{\mathcal{M}}\left(\partial_{z} \hat{v}_{k}\right)^{2} \hat{v}_{k}^{2} d \mathcal{M} \\
= & -\sum_{j, k} \int_{\mathcal{M}}\left(\hat{v}_{j}+\check{v}_{j}\right) \partial_{j} \check{v}_{k} \hat{v}_{k}^{3} d \mathcal{M}-\sum_{k} \int_{\mathcal{M}} w(\hat{\mathbf{v}}+\check{\mathbf{v}}) \partial_{z} \check{v}_{k} \hat{v}_{k}^{3} d \mathcal{M} \\
& -\sum_{j} \frac{1}{\rho_{0}} \int_{\mathcal{M}} \partial_{j} \hat{p}_{s} \hat{v}_{j}^{3} d \mathcal{M}+g \int_{\mathcal{M}} \int_{z}^{0}\left(\beta_{T} \nabla(\hat{T}+\check{T})+\beta_{S} \nabla(\hat{S}+\check{S})\right) d \bar{z} \cdot \hat{\mathbf{v}}^{3} d \mathcal{M} \\
& -f \int_{\mathcal{M}} \mathbf{k} \times(\hat{\mathbf{v}}+\check{\mathbf{v}}) \cdot \hat{\mathbf{v}}^{3} d \mathcal{M}+\int_{\mathcal{M}} F_{\mathbf{v}} \cdot \hat{\mathbf{v}}^{3} d \mathcal{M} \\
:= & J_{1}+J_{2}+J_{3}+J_{4}+J_{5}+J_{6} .
\end{aligned}
$$

Note that we have made use of the cancelation property:

$$
\int_{\mathcal{M}}\left(\mathbf{v}^{\sharp} \cdot \nabla \mathbf{v} \cdot \mathbf{v}^{r}+w\left(\mathbf{v}^{\sharp}\right) \partial_{z} \mathbf{v} \cdot \mathbf{v}^{r}\right) d \mathcal{M}=0,
$$

which holds for any $r \geq 1$ and any vector fields over $\mathcal{M}, \mathbf{v}, \mathbf{v}^{\sharp}$ that are sufficiently smooth and satisfy the boundary conditions (1.4), (1.5), (1.6). Notice moreover that for some constant $\kappa>0$ (depending only on $\left.\mu_{\mathbf{v}}, \nu_{\mathbf{v}}\right)$ we have that

$$
\kappa\left|\nabla_{3} \hat{\mathbf{v}}^{2}\right|^{2} \leq \frac{\mu_{\mathbf{v}}}{2} \sum_{j, k} \int_{\mathcal{M}}\left(\partial_{j} \hat{v}_{k}\right)^{2} \hat{v}_{k}^{2} d \mathcal{M}+\frac{\nu_{\mathbf{v}}}{2} \sum_{k} \int_{\mathcal{M}}\left(\partial_{z} \hat{v}_{k}\right)^{2} \hat{v}_{k}^{2} d \mathcal{M}
$$


where $\nabla_{3}=\left(\partial_{1}, \partial_{2}, \partial_{z}\right)$ is the full three dimensional gradient operator.

Estimates for the Nonlinear Terms $J_{1}, J_{2}$.

We first provide estimates for the inertial terms $J_{1}, J_{2}$ in (4.7). For $J_{1}$ we estimate:

$$
\begin{aligned}
\left|\sum_{k, j} \int_{\mathcal{M}} \hat{v}_{j} \partial_{j} \check{v}_{k} \hat{v}_{k}^{3} d \mathcal{M}\right| & \leq c \sum_{k, j} \int_{\mathcal{M}}\left|\partial_{j} \check{v}_{k}\right|\left(\left|\hat{v}_{k}\right|^{4}+\left|\hat{v}_{j}\right|^{4}\right) d \mathcal{M} \leq c|\nabla \check{\mathbf{v}}|_{L^{6}} \sum_{j}\left(\int_{\mathcal{M}}\left(\left|\hat{v}_{j}\right|^{4}\right)^{6 / 5} d \mathcal{M}\right)^{5 / 6} \\
& \leq c|A \check{U}| \sum_{j}\left(\int_{\mathcal{M}}\left(\hat{v}_{j}^{2}\right)^{12 / 5} d \mathcal{M}\right)^{(5 / 12) \cdot 2} \leq c|A \check{U}|\left(\left|\hat{\mathbf{v}}^{2}\right|^{3 / 4}\left|\nabla_{3}\left(\hat{\mathbf{v}}^{2}\right)\right|^{1 / 4}\right)^{2} \\
& \leq c|A \check{U}||\hat{\mathbf{v}}|_{L^{4}}^{3}\left|\nabla_{3}\left(\hat{\mathbf{v}}^{2}\right)\right|^{1 / 2} \leq\left. c|A \check{U}|^{4 / 3}\left|\hat{\mathbf{v}}_{L^{4}}^{4}+\kappa\right| \nabla_{3}\left(\hat{\mathbf{v}}^{2}\right)\right|^{2}
\end{aligned}
$$

Here we have used the Sobolev embeddings in $\mathbb{R}^{3}$ of $H^{1}$ into $L^{6}$ and of $H^{1 / 4}$ into $L^{5 / 12}$. For $J_{1}$ we also need to estimate

$$
\left|\sum_{k, j} \int_{\mathcal{M}} \check{v}_{j} \partial_{j} \check{v}_{k} \hat{v}_{k}^{3} d \mathcal{M}\right| \leq c|\check{\mathbf{v}}|_{L^{\infty}}|\nabla \check{\mathbf{v}}|_{L^{4}}\left|\hat{\mathbf{v}}^{3}\right|_{L^{4 / 3}} \leq c|A \check{U}|^{2}\left(1+|\hat{\mathbf{v}}|_{L^{4}}^{4}\right)
$$

In summary,

$$
\left|J_{1}\right| \leq c\left(1+|A \check{U}|^{2}\right)\left(1+|\hat{\mathbf{v}}|_{L^{4}}^{4}\right)+\kappa\left|\nabla_{3}\left(\hat{\mathbf{v}}^{2}\right)\right|^{2}
$$

The estimates for $J_{2}$ make use of a preliminary integration by parts (cf. (2.1b))

$$
\begin{aligned}
& -\sum_{k} \int_{\mathcal{M}} w(\hat{\mathbf{v}}+\check{\mathbf{v}}) \partial_{z} \check{v}_{k} \hat{v}_{k}^{3} d \mathcal{M} \\
& \quad=\sum_{j, k} \int_{\mathcal{M}} \int_{z}^{0}\left(\hat{v}_{j}+\check{v}_{j}\right) d \bar{z} \partial_{z j} \check{v}_{k} \hat{v}_{k}^{3} d \mathcal{M}+3 \sum_{j, k} \int_{\mathcal{M}} \int_{z}^{0}\left(\hat{v}_{j}+\check{v}_{j}\right) d \bar{z} \partial_{z} \check{v}_{k} \partial_{j} \hat{v}_{k} \hat{v}_{k}^{2} d \mathcal{M} \\
& \quad:=J_{2,1}+J_{2,2}+J_{2,3}+J_{2,4} .
\end{aligned}
$$

For $J_{2,1}$ we use the embedding of $H^{3 / 4}$ into $L^{4}$ in $\mathbb{R}^{3}$ and find,

$$
\begin{aligned}
\left|J_{2,1}\right| & \leq c|\hat{\mathbf{v}}|_{L^{8}}\left|\nabla \partial_{z} \check{\mathbf{v}}\right|\left|\hat{\mathbf{v}}^{3}\right|_{L^{8 / 3}} \leq c|A \check{U}||\hat{\mathbf{v}}|_{L^{8}}^{4} \leq c|A \check{U}| \sum_{j}\left(\int_{\mathcal{M}}\left(\hat{v}_{j}^{2}\right)^{4}\right)^{(1 / 4) \cdot 2} \\
& \leq c|A \check{U}|\left(\left|\hat{\mathbf{v}}^{2}\right|^{1 / 4}\left|\nabla_{3}\left(\hat{\mathbf{v}}^{2}\right)\right|^{3 / 4}\right)^{2} \leq c|A \check{U}|^{4}|\hat{\mathbf{v}}|_{L^{4}}^{4}+\frac{\kappa}{2}\left|\nabla_{3}\left(\hat{\mathbf{v}}^{2}\right)\right|^{2}
\end{aligned}
$$

For $J_{2,2}$, by making use of Agmon's inequality and that $H^{1 / 2}$ is embedded into $L^{3}$ we infer:

$$
\begin{aligned}
\left|J_{2,2}\right| & \leq|\check{\mathbf{v}}|_{L^{\infty}}\left|\nabla \partial_{z} \check{\mathbf{v}}\right|\left|\mathbf{v}^{3}\right| \leq c|\check{\mathbf{v}}|_{L^{\infty}}\left|\nabla \partial_{z} \check{\mathbf{v}}\right|\left|\hat{\mathbf{v}}^{2}\right|_{L^{3}}^{3 / 2} \leq c|A \check{U}|^{2}\left(\left|\hat{\mathbf{v}}^{2}\right|^{1 / 2}\left|\nabla_{3}\left(\hat{\mathbf{v}}^{2}\right)\right|^{1 / 2}\right)^{3 / 2} \leq c|A \check{U}|^{2}|\hat{\mathbf{v}}|_{L^{4}}^{3 / 2}\left|\nabla_{3}\left(\hat{\mathbf{v}}^{2}\right)\right|^{3 / 4} \\
& \leq c|A \check{U}|^{16 / 5}|\hat{\mathbf{v}}|_{L^{4}}^{12 / 5}+\frac{\kappa}{2}\left|\nabla_{3}\left(\hat{\mathbf{v}}^{2}\right)\right|^{2} \leq c\left(1+|A \check{U}|^{4}\right)\left(1+|\hat{\mathbf{v}}|_{L^{4}}^{4}\right)+\frac{\kappa}{2}\left|\nabla_{3}\left(\hat{\mathbf{v}}^{2}\right)\right|^{2} .
\end{aligned}
$$


$J_{2,3}$ and $J_{2,4}$ seem to require 'anisotropic' type estimates:

$$
\begin{aligned}
\left|J_{2,3}+J_{2,4}\right| & \leq \sum_{j, k} \int_{\mathcal{M}_{0}}\left|\int_{z}^{0}\left(\hat{v}_{j}+\check{v}_{j}\right) d \bar{z}\right|_{L_{z}^{\infty}}\left|\partial_{z} \check{v}_{k}\right|_{L_{z}^{6}}\left|\partial_{j} \hat{v}_{k} \hat{v}_{k}\right|_{L_{z}^{2}}\left|\hat{v}_{k}\right|_{L_{z}^{3}} d \mathcal{M}_{0} \\
& \leq \sum_{j, k} \int_{\mathcal{M}_{0}}\left|\hat{v}_{j}+\check{v}_{j}\right|_{L_{z}^{2}}\left|\partial_{z} \check{v}_{k}\right|_{L_{z}^{6}}\left|\partial_{j} \hat{v}_{k} \hat{v}_{k}\right|_{L_{z}^{2}}\left|\hat{v}_{k}\right|_{L_{z}^{3}} d \mathcal{M}_{0} \\
& \leq \sum_{j, k}\left|\hat{v}_{j}+\check{v}_{j}\right|_{L_{\mathbf{x}}^{12} L_{z}^{2}}\left|\partial_{z} \check{v}_{k}\right|_{L_{\mathbf{x}}^{6} L_{z}^{6}}\left|\partial_{j} \hat{v}_{k} \hat{v}_{k}\right|_{L_{\mathbf{x}}^{2} L_{z}^{2}}\left|\hat{v}_{k}\right|_{L_{\mathbf{x}}^{4} L_{z}^{3}} \\
& \leq c\|U\||A \check{U}|\left(\sum_{j, k} \int_{\mathcal{M}}\left(\partial_{j} \hat{v}_{k}\right)^{2} \hat{v}_{k}^{2} d \mathcal{M}\right)^{1 / 2}|\hat{\mathbf{v}}|_{L^{4}} \\
& \leq c\|U\|^{2}|A \check{U}|^{2}\left(1+|\hat{\mathbf{v}}|_{L^{4}}^{4}\right)+\frac{1}{2} \mu_{\mathbf{v}} \sum_{j, k} \int_{\mathcal{M}}\left(\partial_{j} \hat{v}_{k}\right)^{2} \hat{v}_{k}^{2} d \mathcal{M} .
\end{aligned}
$$

Note that we made use of Remark 2.1, (2.2) with $q=12$ in order to estimate $\left|\hat{v}_{j}+\check{v}_{j}\right|_{L_{z}^{2} L_{x}^{12}}=\left|v_{j}\right|_{L_{z}^{2} L_{x}^{12}} \leq c\|U\|$. Summarizing the above estimates and taking advantage of the observation (4.9) we conclude that

$$
\left|J_{2}\right| \leq c\left(1+\|U\|^{2}\right)\left(1+|A \check{U}|^{4}\right)\left(1+|\hat{\mathbf{v}}|_{L^{4}}^{4}\right)+\mu_{\mathbf{v}} \sum_{j, k} \int_{\mathcal{M}}\left(\partial_{j} \hat{v}_{k}\right)^{2} \hat{v}_{k}^{2} d \mathcal{M}+\nu_{\mathbf{v}} \sum_{j, k} \int_{\mathcal{M}}\left(\partial_{z} \hat{v}_{k}\right)^{2} \hat{v}_{k}^{2} d \mathcal{M}
$$

\section{Estimates for the Pressure Term $J_{3}$}

We next attend to the term $J_{3}$. Using the fact that the pressure term, $p_{s}$, depends only on the horizontal variable $\mathbf{x}$ we find

$$
\left|J_{3}\right|=\left|\sum_{j} \frac{1}{\rho_{0}} \int_{\mathcal{M}_{0}} \partial_{j} \hat{p}_{s} \int_{-h}^{0} \hat{v}_{j}^{3} d z d \mathcal{M}_{0}\right| \leq c\left|\nabla p_{s}\right|_{L_{x}^{4 / 3}}\left|\int_{-h}^{0} \hat{\mathbf{v}}^{3} d z\right|_{L_{\mathbf{x}}^{4}} .
$$

By applying the Sobolev embedding in $D=2$ that $W^{4 / 3,1}$ is embedded in $L^{4}\left(\mathcal{M}_{0}\right)$ we have

$$
\begin{aligned}
\left|\int_{-h}^{0} \hat{\mathbf{v}}^{3} d z\right|_{L_{x}^{4}} & \leq c \sum_{j, k}\left|\partial_{j} \int_{-h}^{0} \hat{v}_{k}^{3} d z\right|_{L_{x}^{4 / 3}} \leq c \sum_{j, k}\left|\int_{-h}^{0}\left(\partial_{j} \hat{v}_{k} \hat{v}_{k}\right) \hat{v}_{k} d z\right|_{L_{x}^{4 / 3}} \\
& \leq c \sum_{j, k}\left(\int_{\mathcal{M}_{0}}\left(\int_{-h}^{0}\left(\partial_{j} \hat{v}_{k}\right)^{2} \hat{v}_{k}^{2} d z\right)^{2 / 3}\left(\int_{-h}^{0} \hat{v}_{k}^{2} d z\right)^{2 / 3} d \mathcal{M}_{0}\right)^{3 / 4} \\
& \leq c|\hat{\mathbf{v}}|_{L^{4}} \sum_{j, k}\left(\int_{\mathcal{M}}\left(\partial_{j} \hat{v}_{k}\right)^{2} \hat{v}_{k}^{2} d \mathcal{M}\right)^{1 / 2} .
\end{aligned}
$$

Combining these estimates and using Young's inequality we conclude that:

$$
\left|J_{3}\right| \leq c\left|\nabla p_{s}\right|_{L_{x}^{4 / 3}}^{2}|\hat{\mathbf{v}}|_{L^{4}}^{2}+\mu_{\mathbf{v}} \sum_{j, k} \int_{\mathcal{M}}\left(\partial_{j} \hat{v}_{k}\right)^{2} \hat{v}_{k}^{2} d \mathcal{M}
$$

Remark 4.2. Note that it is at precisely this stage in the estimates that we see the crucial role played by the two dimensional spatial dependence of the surface pressure $p_{s}$. This insight concerning the importance of the lower dimensional pressure is the key to the recent breakthroughs for global existence in [CT07, Kob06, Kob07, KZ07]. In the present study we follow the approach in [KZ07] which treats the pressure explicitly via bounds on the pressure for the Stokes equation found in [SvW86] and reproduced below in Proposition 6.1 for the convenience of the reader. 
In order to estimate the term $\left|\nabla p_{s}\right|_{L^{4 / 3}}$ appearing in (4.12) we now apply Proposition 6.1 below. To this end we average (4.5) in the vertical direction. Define the operator $\mathfrak{A}(\mathbf{v})=\frac{1}{h} \int_{-h}^{0} \mathbf{v} d z$ and let $\mathbf{q}=\rho_{0} \mathfrak{A}(\hat{\mathbf{v}})$. By applying $\mathfrak{A}$ to (4.5) we find that $\mathbf{q}$ satisfies the following Stokes system over $\mathcal{M}_{0} \subset \mathbb{R}^{2}$ :

$$
\begin{gathered}
\partial_{t} \mathbf{q}-\mu_{\mathbf{v}} \Delta \mathbf{q}+\nabla \hat{p}_{s}=G(U), \\
\nabla \cdot \mathbf{q}=0, \quad \mathbf{q}_{\mid \partial \mathcal{M}_{0}}=0,
\end{gathered}
$$

where, for $\mathbf{v}=\hat{\mathbf{v}}+\check{\mathbf{v}}$,

$$
\begin{aligned}
G(U) & =G(\mathbf{v}, T, S)=G_{1}(U)+G_{2}(U) \\
& :=-\rho_{0} \mathfrak{A}((\mathbf{v} \cdot \nabla) \mathbf{v}+(\nabla \cdot \mathbf{v}) \mathbf{v})+\rho_{0} \mathfrak{A}\left(g \int_{z}^{0}\left(\beta_{T} \nabla T+\beta_{S} \nabla S\right) d \bar{z}-f \mathbf{k} \times \mathbf{v}+F_{\mathbf{v}}\right) .
\end{aligned}
$$

Note that, using (1.2c) and taking into account the boundary conditions (1.4), (1.5), $\mathfrak{A}\left(w(\mathbf{v}) \partial_{z} \mathbf{v}\right)=\mathfrak{A}((\nabla$. $\mathbf{v}) \mathbf{v})$ and $\mathfrak{A}\left(\partial_{z z} \hat{\mathbf{v}}\right)=0$.

Making use of Proposition 6.1 below, we find, for any $0<t<\infty$, and for any pair of stopping times $\tau_{a}$, $\tau_{b}$, with $0 \leq \tau_{a} \leq \tau_{b} \leq t \wedge \xi$ that

$$
\int_{\tau_{a}}^{\tau_{b}}\left|\nabla \hat{p}_{s}\right|_{L_{\mathbf{x}}^{4 / 3}}^{2} d t^{\prime} \leq c\left(\left\|\mathbf{q}\left(\tau_{a}\right)\right\|^{2}+\int_{\tau_{a}}^{\tau_{b}}|G(U)|_{L_{\mathbf{x}}^{4 / 3}}^{2} d t^{\prime}\right) .
$$

Observe that $\left\|\mathbf{q}\left(\tau_{a}\right)\right\| \leq c\left\|\hat{\mathbf{v}}\left(\tau_{a}\right)\right\|$ and that

$$
\int_{\tau_{a}}^{\tau_{b}}\left|G_{2}(U)\right|_{L_{\mathbf{x}}^{4 / 3}}^{2} d t^{\prime} \leq c \int_{\tau_{a}}^{\tau_{b}}\left|G_{2}(U)\right|^{2} d t^{\prime} \leq c \int_{\tau_{a}}^{\tau_{b}}\left(\|U\|^{2}+|F|^{2}\right) d t^{\prime} .
$$

On the other hand,

$$
\begin{aligned}
\int_{\tau_{a}}^{\tau_{b}}\left|G_{1}(U)\right|_{L_{\mathbf{x}}^{4 / 3}}^{2} d t^{\prime} & \leq c \int_{\tau_{a}}^{\tau_{b}}|(\mathbf{v} \cdot \nabla) \mathbf{v}+(\nabla \cdot \mathbf{v}) \mathbf{v}|_{L^{4 / 3}}^{2} d t^{\prime} \\
& \leq c \int_{\tau_{a}}^{\tau_{b}}\left(\int_{\mathcal{M}}|\mathbf{v}|^{4 / 3}|\nabla \mathbf{v}|^{4 / 3} d \mathcal{M}\right)^{3 / 2} d t^{\prime} \leq c \int_{\tau_{a}}^{\tau_{b}}|\mathbf{v}|_{L^{4}}^{2}\|\mathbf{v}\|^{2} d t^{\prime}
\end{aligned}
$$

In conclusion:

$$
\int_{\tau_{a}}^{\tau_{b}}\left|\nabla \hat{p}_{s}\right|_{L_{\mathbf{x}}^{4 / 3}}^{2} d t^{\prime} \leq c\left(\left\|\hat{\mathbf{v}}\left(\tau_{a}\right)\right\|^{2}+\int_{\tau_{a}}^{\tau_{b}}\left(\left(1+|\mathbf{v}|_{L^{4}}^{2}\right)\|U\|^{2}+|F|^{2}\right) d t^{\prime}\right) .
$$

Finally, combining (4.14) and (4.12) we find, for any pair of stopping times $0 \leq \tau_{a} \leq \tau_{b} \leq t \wedge \xi$,

$$
\begin{aligned}
\int_{\tau_{a}}^{\tau_{b}}\left|J_{3}\right| d t^{\prime} \leq & \frac{1}{2} \sup _{t^{\prime} \in\left[\tau_{a}, \tau_{b}\right]}|\hat{\mathbf{v}}|_{L^{4}}^{4}+\mu_{\mathbf{v}} \int_{\tau_{a}}^{\tau_{b}} \sum_{j, k} \int_{\mathcal{M}}\left(\partial_{j} \hat{v}_{k}\right)^{2} \hat{v}_{k}^{2} d \mathcal{M} d t^{\prime} \\
& +c\left(\left\|\hat{\mathbf{v}}\left(\tau_{a}\right)\right\|^{4}+\left(1+\sup _{t^{\prime} \in\left[\tau_{a}, \tau_{b}\right]}\left(|\hat{\mathbf{v}}|_{L^{4}}^{4}+|\check{\mathbf{v}}|_{L^{4}}^{4}\right) \int_{\tau_{a}}^{\tau_{b}}\left(\|U\|^{2}+|F|^{2}\right) d t^{\prime}\right) .\right.
\end{aligned}
$$

Estimates for the Lower Order Terms $J_{4}, J_{5}, J_{6}$

To estimate the term $J_{4}$ we use the embedding of $L^{3}$ into $H^{1 / 2}$ in $2 D$ and we observe that

$$
\begin{aligned}
\left|J_{4}\right| & \leq c\|U\| \sum_{k}\left(\int_{\mathcal{M}}\left(\hat{v}_{k}^{2}\right)^{3} d \mathcal{M}\right)^{1 / 3 \cdot 3 / 2} \leq c\|U\|\left(\left|\hat{\mathbf{v}}^{2}\right|^{1 / 2}\left|\nabla_{3} \hat{\mathbf{v}}^{2}\right|^{1 / 2}\right)^{3 / 2} \\
& \leq \kappa\left|\nabla_{3} \hat{\mathbf{v}}^{2}\right|^{2}+c\left(1+\|U\|^{2}\right)\left(1+|\hat{\mathbf{v}}|_{L^{4}}^{4}\right) .
\end{aligned}
$$


The estimate for the Coriolis term $J_{5}$ is direct

$$
\left|J_{5}\right| \leq c|\mathbf{v}|_{L^{4}}|\hat{\mathbf{v}}|_{L^{4}}^{3} \leq c\|U\|\left(1+|\hat{\mathbf{v}}|_{L^{4}}^{4}\right) .
$$

Finally for $J_{6}$ we find

$$
\left|J_{6}\right| \leq\left|F_{\mathbf{v}}\right|_{L^{4}}|\hat{\mathbf{v}}|_{L^{4}}^{3} \leq c\left(|F|_{L^{4}}^{2}+1\right)\left(|\hat{\mathbf{v}}|_{L^{4}}^{4}+1\right) .
$$

Integrating (4.7) in time from $\tau_{a}$ to $\tau_{b}$ and then putting together the estimates (4.9) (4.10), (4.11), (4.15), (4.16), (4.17), (4.18) we find that for any pair of stopping times $\tau_{a}$, $\tau_{b}$ with $0 \leq \tau_{a} \leq \tau_{b} \leq t \wedge \xi$

$$
\sup _{t^{\prime} \in\left[\tau_{a}, \tau_{b}\right]}|\hat{\mathbf{v}}|_{L^{4}}^{4} \leq c\left\|\hat{\mathbf{v}}\left(\tau_{a}\right)\right\|^{4}+c\left(1+\sup _{t^{\prime} \in\left[\tau_{a}, \tau_{b}\right]}|\hat{\mathbf{v}}|_{L^{4}}^{4}+\sup _{t^{\prime} \in[0, t \wedge \xi]}|\check{\mathbf{v}}|_{L^{4}}^{4}\right) \int_{\tau_{a}}^{\tau_{b}} H\left(t^{\prime}\right) d t^{\prime},
$$

where

$$
H\left(t^{\prime}\right):=\left(1+\left\|U\left(t^{\prime}\right)\right\|^{2}\right)\left(1+\left|A \check{U}\left(t^{\prime}\right)\right|^{4}\right)+\left|F\left(t^{\prime}\right)\right|_{L^{4}}^{2}
$$

We finally apply the version of Gronwall's lemma in Proposition 6.2 below with $p=2, X\left(t^{\prime}\right)=\left|\hat{\mathbf{v}}\left(t^{\prime}\right)\right|_{L^{4}}^{4}$, $f\left(t^{\prime}\right)=c\left\|\hat{\mathbf{v}}\left(t^{\prime}\right)\right\|^{2}, g\left(t^{\prime}\right)=c H\left(t^{\prime}\right)$ and $h\left(t^{\prime}\right)=c\left(1+\sup _{t^{\prime} \in[0, t \wedge \xi]}|\check{\mathbf{v}}|_{L^{4}}^{4}\right) H\left(t^{\prime}\right)$. Observe that, as a consequence of Theorem 3.1 and Proposition 4.2 it is clear that $\hat{U}=U-\check{U} \in C([0, t \wedge \xi) ; V)$. It therefore follows directly that $X \in C([0, t \wedge \xi))$ and that $f$ is continuous at $t=0$. Also, due to Theorem 3.1, Proposition 4.2 and the standing assumption (2.10), we easily infer that $f, g, h \in L^{1}([0, t \wedge \xi])$. Thus by, Proposition 6.2 , we conclude that (4.6) holds and Proposition 4.3 is now proved.

Proof of Proposition 4.1 (conclusion). Combining (4.6) with (4.3) we infer (4.1). By the definition of $\tau_{K}^{(1)}$, (3.4), it follows directly from (4.1) that $\lim _{K \uparrow \infty} \tau_{K}^{(1)}=\infty$, this completes the proof of Proposition 4.1.

\section{$5 \quad$ Vertical Gradient Estimates}

In this section we will show that $\lim _{K \uparrow \infty} \tau_{K}^{(2)}=+\infty$, with $\tau_{K}^{(2)}$ defined in (3.4). Recall that, in view of proving Theorem 3.2 and hence establishing the main result of the article, we need to show that $\lim _{K \uparrow \infty} \tau_{K}=$ $+\infty$, where $\tau_{K}=\tau_{K}^{(1)} \wedge \tau_{K}^{(2)}$ is given by (3.4). Since we have already shown in the previous section that $\lim _{K \uparrow \infty} \tau_{K}^{(1)}=+\infty$ we now need to show that $\lim _{K \uparrow \infty} \tau_{K}^{(2)}=+\infty$ which essentially amounts to deriving suitable bounds for $\partial_{z} U$ in $L^{2}(\mathcal{M})$.

Proposition 5.1. Assume the conditions set out in Theorem 2.1 and suppose that $(U, \xi)$ is the corresponding strong pathwise solution of (1.2). Then, for any $t>0$,

$$
\sup _{t^{\prime} \in[\xi \wedge t]}\left|\partial_{z} U\right|^{2}+\int_{0}^{\xi \wedge t}\left\|\partial_{z} U\right\|^{2} d t^{\prime}<\infty .
$$

Hence, defining $\tau_{K}^{(2)}$ according to (3.4), $\lim _{K \uparrow \infty} \tau_{K}^{(2)}=\infty$.

The bound (5.1) and hence Proposition 5.1 follows directly from (5.4) and (5.18) which are established in Corollary 5.1 and Proposition 5.3 respectively below. For the analysis we make use of several additional stopping times:

$$
\begin{aligned}
\tau_{K}^{\partial_{z} \mathbf{v}} & :=\inf _{t \geq 0}\left\{\sup _{t^{\prime} \in[0, t \wedge \xi]}\left|\partial_{z} \mathbf{v}\left(t^{\prime}\right)\right|^{2}+\int_{0}^{t \wedge \xi}\left\|\partial_{z} \mathbf{v}\left(t^{\prime}\right)\right\|^{2} \geq K\right\}=\sup _{t \geq 0}\left\{\sup _{t^{\prime} \in[0, t \wedge \xi]}\left|\partial_{z} \mathbf{v}\left(t^{\prime}\right)\right|^{2}+\int_{0}^{t \wedge \xi}\left\|\partial_{z} \mathbf{v}\left(t^{\prime}\right)\right\|^{2} \leq K\right\} \\
\tau_{K}^{T, S} & :=\inf _{t \geq 0}\left\{\sup _{t^{\prime} \in[0, t \wedge \xi]}\left(\left|T\left(t^{\prime}\right)\right|_{L^{4}}^{4}+\left|S\left(t^{\prime}\right)\right|_{L^{4}}^{4}\right) \geq K\right\}=\sup _{t \geq 0}\left\{\sup _{t^{\prime} \in[0, t \wedge \xi]}\left(\left|T\left(t^{\prime}\right)\right|_{L^{4}}^{4}+\left|S\left(t^{\prime}\right)\right|_{L^{4}}^{4}\right) \leq K\right\} \\
\sigma_{K} & :=\tau_{K}^{\partial_{z} \mathbf{v}} \wedge \tau_{K}^{T, S} \wedge \tau_{K}^{(1)} .
\end{aligned}
$$


Here $\tau_{K}^{(1)}$ is defined as in (3.4). We first tackle $\partial_{z} \mathbf{v}$ in $L^{2}$. From these estimates along with those carried out above for $\mathbf{v}$ in $L^{4}(\mathcal{M})$ we infer that $\lim _{K \uparrow \infty} \tau_{K}^{\partial_{z} \mathbf{v}}=\infty$. We next would like to estimate $\partial_{z} T$ and $\partial_{z} S$ in $L^{2}(\mathcal{M})$. However, in order to be able to treat the nonlinear terms which arise (see, for example, $(5.20)$ below) we first must estimate $T$ and $S$ in $L^{4}(\mathcal{M})$. This is the reason for the second collection of stopping times, $\tau_{K}^{T, S}$, are defined in (5.2). In this case, in contrast to the momentum equations above, there are no pressure terms which means that we can perform these $L^{4}$ estimates in the original variables by means of the Itō lemma. With these estimates we deduce that $\lim _{K \uparrow \infty} \sigma_{K}=\infty$ which allows us to finally establish the desired bounds for $\partial_{z} T$ and $\partial_{z} S$, (5.18), in Proposition 5.3. Having now established (5.1), we thus immediately infer that $\lim _{K \uparrow \infty} \tau_{K}^{(2)}=\infty$, as desired for Theorem 3.2.

\subsection{Estimates for the Momentum Equations}

Proposition 5.2. Assume the conditions set out in Theorem 2.1 and suppose that $(U, \xi)$ is the corresponding strong pathwise solution of (1.2). Define $\tau_{K}^{(1)}$ as in (3.4). Then, for any $t>0$,

$$
\mathbb{E}\left(\sup _{t^{\prime} \in\left[0, \tau_{K}^{(1)} \wedge \xi \wedge t\right]}\left|\partial_{z} \mathbf{v}\right|^{2}+\int_{0}^{\tau_{K}^{(1)} \wedge \xi \wedge t}\left\|\partial_{z} \mathbf{v}\right\|^{2} d t^{\prime}\right)<\infty .
$$

From this proposition, proven immediately below, we obtain the following

Corollary 5.1. Assume that the conditions set out in Theorem 2.1 hold and that $(U, \xi)$ is the resulting strong, pathwise solution of (1.2). Then for, any $t>0$,

$$
\sup _{t^{\prime} \in[\xi \wedge t]}\left|\partial_{z} \mathbf{v}\right|^{2}+\int_{0}^{\xi \wedge t}\left\|\partial_{z} \mathbf{v}\right\|^{2} d t^{\prime}<\infty \quad \text { a.s. }
$$

Moreover, defining the stopping times $\tau_{K}^{\partial_{z} \mathbf{v}}$ according to (5.2) we have that $\lim _{K \uparrow \infty} \tau_{K}^{\partial_{z} \mathbf{v}}=\infty$.

Proof of Proposition 5.2: We apply $\partial_{z}$ to (2.1a) to infer an evolution equation for $\partial_{z} \mathbf{v}$ :

$$
\begin{aligned}
d \partial_{z} \mathbf{v} & +\left(\partial_{z}\left[(\mathbf{v} \cdot \nabla) \mathbf{v}+w(\mathbf{v}) \partial_{z} \mathbf{v}\right]+g \partial_{z}\left[\beta_{T} \nabla T+\beta_{S} \nabla S\right]+f \mathbf{k} \times\left(\partial_{z} \mathbf{v}\right)\right) d t \\
& -\left(\mu_{\mathbf{v}} \Delta \partial_{z} \mathbf{v}+\nu_{\mathbf{v}} \partial_{z z z} \mathbf{v}\right) d t=\partial_{z} F_{\mathbf{v}} d t+\partial_{z} \sigma_{\mathbf{v}}(\mathbf{v}, T, S) d W
\end{aligned}
$$

Note that, since $\hat{p}_{s}$ is independent of $z$, the pressure term does not appear above. After an application of the Itō formula to this system we find

$$
\begin{aligned}
d\left|\partial_{z} \mathbf{v}\right|^{2}+2\left\|\partial_{z} \mathbf{v}\right\|^{2} d t= & -2 \int_{\mathcal{M}}\left(\partial_{z}\left[(\mathbf{v} \cdot \nabla) \mathbf{v}+w(\mathbf{v}) \partial_{z} \mathbf{v}\right]+g \partial_{z}\left[\beta_{T} \nabla T+\beta_{S} \nabla S\right]\right) \cdot \partial_{z} \mathbf{v} d \mathcal{M} d t \\
& +2\left(\partial_{z} F_{\mathbf{v}}, \partial_{z} \mathbf{v}\right) d t+\left|\partial_{z} \sigma_{\mathbf{v}}(\mathbf{v}, T, S)\right|_{L_{2}(\mathfrak{U}, H)}^{2} d t+2\left(\partial_{z} \sigma_{\mathbf{v}}(\mathbf{v}, T, S), \partial_{z} \mathbf{v}\right) d W \\
= & \left(J_{1}^{z}+J_{2}^{z}+J_{3}^{z}+J_{4}^{z}+J_{5}^{z}\right) d t+J_{6}^{z} d W .
\end{aligned}
$$

Here we have made use of the boundary conditions (1.4), (1.5), (1.6) in order to infer $\left(-\left(\mu_{\mathbf{v}} \Delta \partial_{z} \mathbf{v}+\right.\right.$ $\left.\left.\nu_{\mathbf{v}} \partial_{z z z} \mathbf{v}\right), \partial_{z} \mathbf{v}\right)=\left\|\partial_{z} \mathbf{v}\right\|^{2}$. Note also that there is a cancelation in the 'Coriolis term' $\left(k \times \partial_{z} \mathbf{v}, \partial_{z} \mathbf{v}\right)=0$.

We begin by treating the nonlinear terms. For $J_{1}^{z}$, an integration by parts reveals

$$
\begin{aligned}
J_{1}^{z} & =-\sum_{j, k} \int_{\mathcal{M}} \partial_{z} v_{j} \partial_{j} v_{k} \partial_{z} v_{k} d \mathcal{M}-\sum_{j, k} \int_{\mathcal{M}} v_{j} \partial_{z} \partial_{j} v_{k} \partial_{z} v_{k} d \mathcal{M} \\
& =\sum_{j, k} \int_{\mathcal{M}} \partial_{j} \partial_{z} v_{j} v_{k} \partial_{z} v_{k} d \mathcal{M}+\sum_{j, k} \int_{\mathcal{M}} \partial_{z} v_{j} v_{k} \partial_{j} \partial_{z} v_{k} d \mathcal{M}-\sum_{j, k} \int_{\mathcal{M}} v_{j} \partial_{z} \partial_{j} v_{k} \partial_{z} v_{k} d \mathcal{M} .
\end{aligned}
$$


Hence with direct estimates using Holder's inequality and the Sobolev embedding of $H^{3 / 4}$ into $L^{4}(\mathcal{M})$

$$
\left|J_{1}^{z}\right| \leq c\left\|\partial_{z} \mathbf{v}\right\||\mathbf{v}|_{L^{4}}\left|\partial_{z} \mathbf{v}\right|_{L^{4}} \leq\left. c\left\|\partial_{z} \mathbf{v}\right\|\right|^{7 / 4}|\mathbf{v}|_{L^{4}}\left|\partial_{z} \mathbf{v}\right|^{1 / 4} \leq c|\mathbf{v}|_{L^{4}}^{8}\left|\partial_{z} \mathbf{v}\right|^{2}+\frac{1}{3}\left\|\partial_{z} \mathbf{v}\right\|^{2}
$$

For the second portion, $J_{2}^{z}$, of the nonlinear term we have:

$$
\begin{aligned}
J_{2}^{z} & =\int_{\mathcal{M}} w(\mathbf{v}) \partial_{z} \mathbf{v} \cdot \partial_{z z} \mathbf{v} d \mathcal{M}=\frac{1}{2} \int_{\mathcal{M}} w(\mathbf{v}) \partial_{z}\left(\partial_{z} \mathbf{v} \cdot \partial_{z} \mathbf{v}\right) d \mathcal{M} \\
& =\frac{1}{2} \int_{\mathcal{M}} \nabla \cdot \mathbf{v} \partial_{z} \mathbf{v} \cdot \partial_{z} \mathbf{v} d \mathcal{M}=-\sum_{j} \int_{\mathcal{M}} v_{j} \partial_{j} \partial_{z} \mathbf{v} \cdot \partial_{z} \mathbf{v} d \mathcal{M}
\end{aligned}
$$

and hence, as for $J_{1}^{z}$ we may estimate

$$
\left|J_{2}^{z}\right| \leq c|\mathbf{v}|_{L^{4}}^{8}\left|\partial_{z} \mathbf{v}\right|^{2}+\frac{1}{3}\left\|\partial_{z} \mathbf{v}\right\|^{2}
$$

Direct estimates and using the assumption (2.13) lead to

$$
\left|J_{3}^{z}\right|+\left|J_{4}^{z}\right|+\left|J_{5}^{z}\right| \leq c\left(1+\|U\|^{2}+|F|^{2}\right)+\frac{1}{3}\left\|\partial_{z} \mathbf{v}\right\|^{2}
$$

On other other hand, applying the Burkholder-Davis-Gundy inequality, (2.11), and again using (2.13) we find that for $0 \leq \tau_{a} \leq \tau_{b} \leq t \wedge \xi \wedge \tau_{K}^{(1)}$

$$
\begin{aligned}
& \mathbb{E} \sup _{t^{\prime} \in\left[\tau_{a}, \tau_{b}\right]}\left|\int_{\tau_{a}}^{t^{\prime}} J_{6}^{z} d W\right| \\
& \quad \leq c \mathbb{E}\left(\int_{\tau_{a}}^{\tau_{b}}\left(\partial_{z} \sigma_{\mathbf{v}}(U), \partial_{z} \mathbf{v}\right)^{2} d t^{\prime}\right)^{1 / 2} \leq c \mathbb{E}\left(\int_{\tau_{a}}^{\tau_{b}}\left|\sigma_{\mathbf{v}}(U)\right|_{L_{2}(\mathfrak{U}, V)}^{2}\left|\partial_{z} \mathbf{v}\right|^{2} d t^{\prime}\right)^{1 / 2} \\
& \quad \leq c \mathbb{E}\left[\sup _{t^{\prime} \in\left[\tau_{a}, \tau_{b}\right]}\left|\partial_{z} \mathbf{v}\right|\left(\int_{\tau_{a}}^{\tau_{b}}\left(1+\|U\|^{2}\right) d t^{\prime}\right)^{1 / 2}\right] \leq \frac{1}{2} \mathbb{E}_{t^{\prime} \in\left[\tau_{a}, \tau_{b}\right]}\left|\partial_{z} \mathbf{v}\right|^{2}+c \mathbb{E} \int_{\tau_{a}}^{\tau_{b}}\left(1+\|U\|^{2}\right) d t^{\prime}
\end{aligned}
$$

Gathering the estimates (5.7), (5.8), (5.9), (5.10) we apply the stochastic Gronwall Lemma, Proposition 6.3 (from [GHZ09, Lemma 5.3]) and infer (5.3). This completes the proof of Proposition 5.2.

\section{$5.2 L^{4}(\mathcal{M})$ estimates for the Temperature and Salinity Equations}

We turn to the estimates for the temperature and the salinity, beginning with the estimates in $L^{4}(\mathcal{M})$.

Lemma 5.1. Suppose the standing conditions as in Theorem 2.1 are satisfied and let $(U, \xi)$ be the maximal strong solution of (2.14). Define $\tau_{K}^{W}$ as in (3.3). Then, for any $t>0$,

$$
\mathbb{E}\left(\sup _{t^{\prime} \in\left[0, t \wedge \tau_{K}^{W} \wedge \xi\right]}\left(|T|_{L^{4}}^{4}+|S|_{L^{4}}^{4}\right)\right)<\infty
$$

Moreover,

$$
\sup _{t^{\prime} \in[0, t \wedge \xi]}\left(|T|_{L^{4}}^{4}+|S|_{L^{4}}^{4}\right)<\infty . \quad \text { a.s. }
$$

With $\tau_{K}^{T, S}$ defined as in (5.2), we have that $\lim _{K \uparrow \infty} \tau_{K}^{T, S}=\infty$. 
Proof. We carry out the estimates for the temperature $T$. Those for the salinity $S$ are identical. In order to find an evolution equation for $|T|_{L^{4}}^{4}$ we may proceed similarly to e.g. [Kry99, MR01]. We apply the Itō lemma to (2.1c) pointwise for almost every $(\mathbf{x}, z) \in \mathcal{M}$. This is justified given the regularity already established for $T$ in Theorem 3.1. We then integrate the resulting system over $\mathcal{M}$, apply the stochastic Fubini theorem (see [DPZ92]) and find that

$$
\begin{aligned}
d|T|_{L^{4}}^{4} & +\left(12 \mu_{T} \sum_{j} \int_{\mathcal{M}}\left(\partial_{j} T\right)^{2} T^{2} d \mathcal{M}+12 \nu_{T} \int_{\mathcal{M}}\left(\partial_{z} T\right)^{2} T^{2} d \mathcal{M}\right) d t \\
& =4 \int_{\mathcal{M}} F_{T} T^{3} d \mathcal{M} d t+6 \sum_{l \geq 1} \int_{\mathcal{M}} \sigma_{T}(U)_{l}^{2} T^{2} d \mathcal{M} d t+4 \sum_{l \geq 1} \int_{\mathcal{M}} \sigma_{T}(U)_{l} T^{3} d \mathcal{M} d W_{l} .
\end{aligned}
$$

Note also that we have once again used the cancelation properties of the nonlinear portion of (2.1c) (cf. (4.8)) in inferring (5.13).

The external body forcing term is estimated as above (cf. (4.18)):

$$
\left|\int_{\mathcal{M}} F_{T} T^{3} d \mathcal{M}\right| \leq c\left(\left|F_{T}\right|_{L^{4}}^{2}+1\right)\left(|T|_{L^{4}}^{4}+1\right) .
$$

For the Itō correction term, using (2.13), we find

$$
\begin{aligned}
\left|\sum_{l} \int_{\mathcal{M}} \sigma_{T}(U)_{l}^{2} T^{2} d \mathcal{M}\right| & \leq c \sum_{l}\left|\sigma_{T}(U)_{l}\right|_{L^{4}}^{2}|T|_{L^{4}}^{2} \\
& \leq c\left|\sigma_{T}(U)\right|_{L_{2}(\mathfrak{U}, V)}^{2}|T|_{L^{4}}^{2} \\
& \leq c\left(1+\|U\|^{2}\right)\left(1+|T|_{L^{4}}^{4}\right) .
\end{aligned}
$$

With an application of the BDG inequality, (2.11), we find

$$
\begin{aligned}
\mathbb{E} \sup _{t \in\left[\tau_{a}, \tau_{b}\right]}\left|\int_{\tau_{a}}^{t} \sum_{l} \int_{\mathcal{M}} \sigma_{T}(U)_{l} T^{3} d \mathcal{M} d W_{l}\right| & \leq c \mathbb{E}\left(\int_{\tau_{a}}^{\tau_{b}} \sum_{l}\left(\int_{\mathcal{M}} \sigma_{T}(U)_{l} T^{3} d \mathcal{M}\right)^{2} d t\right)^{1 / 2} \\
& \leq c \mathbb{E}\left(\int_{\tau_{a}}^{\tau_{b}} \sum_{l}\left|\sigma_{T}(U)_{l}\right|_{L^{4}}^{2}|T|_{L^{4}}^{6} d t\right)^{1 / 2} \\
& \leq c \mathbb{E}\left(\int_{\tau_{a}}^{\tau_{b}}\|\sigma(U)\|_{L_{2}(\mathfrak{U}, V)}^{2}|T|_{L^{4}}^{6} d t\right)^{1 / 2} \\
& \leq c \mathbb{E} \sup _{t \in\left[\tau_{a}, \tau_{b}\right]}|T|_{L^{4}}^{3}\left(\int_{\tau_{a}}^{\tau_{b}}\left(1+\|U\|^{2}\right)|T|_{L^{4}}^{2} d t\right)^{1 / 2} \\
& \leq \frac{1}{2} \mathbb{E} \sup _{t \in\left[\tau_{a}, \tau_{b}\right]}|T|_{L^{4}}^{4}+c \mathbb{E} \int_{\tau_{a}}^{\tau_{b}}\left(1+\|U\|^{2}\right)\left(1+|T|_{L^{4}}^{4}\right) d t
\end{aligned}
$$

Here, similarly to (5.10) above, $\tau_{a}, \tau_{b}$ may be any stopping time such that $0 \leq \tau_{a} \leq \tau_{b} \leq t \wedge \tau_{K}^{W} \wedge \xi$. Combining the estimates (5.14), (5.15), (5.16) with (5.13) we apply the stochastic Gronwall lemma, Proposition 6.3, to infer (5.11). Now, due to Theorem 3.1, $\lim _{K \uparrow \infty} \tau_{K}^{W}=\infty$. With this observation (5.12) and thus that $\lim _{K \uparrow \infty} \tau_{K}^{T, S}=\infty$ follow directly from (5.11). This completes the proof of Lemma 5.1

The final step is now to carry out the estimates for $\partial_{z} T$ and $\partial_{z} S$ in $L^{2}(\mathcal{M})$ : 
Proposition 5.3. Assume the conditions of Theorem 2.1 consider $(U, \xi)$ the maximal strong solution of (2.14). Take $\sigma_{K}$ according to (5.2). Then, for any $t>0$,

$$
\mathbb{E}\left(\sup _{t \in\left[0, t \wedge \sigma_{K} \wedge \xi\right]}\left(\left|\partial_{z} T\right|^{2}+\left|\partial_{z} S\right|^{2}\right)+\int_{0}^{t \wedge \sigma_{K} \wedge \xi}\left(\left\|\partial_{z} T\right\|^{2}+\left\|\partial_{z} S\right\|^{2}\right) d t^{\prime}\right)<\infty .
$$

Moreover, up to a set of measure zero,

$$
\sup _{t \in[0, t \wedge \xi]}\left(\left|\partial_{z} T\right|^{2}+\left|\partial_{z} S\right|^{2}\right)+\int_{0}^{t \wedge \xi}\left(\left\|\partial_{z} T\right\|^{2}+\left\|\partial_{z} S\right\|^{2}\right) d t^{\prime}<\infty .
$$

Proof. As above, in Lemma 5.1 we provide the estimates for $T$; those for $S$ are identical. To this end we apply $\partial_{z}$ to $(2.1 \mathrm{c})$ and find:

$$
d \partial_{z} T+\partial_{z}\left[(\mathbf{v} \cdot \nabla) T+w(\mathbf{v}) \partial_{z} T\right] d t-\left(\mu_{T} \Delta \partial_{z} T+\nu_{T} \partial_{z z} \partial_{z} T\right) d t=\partial_{z} F_{T} d t+\partial_{z} \sigma_{T}(\mathbf{v}, T, S) d W .
$$

An application of the Itō formula then reveals:

$$
\begin{aligned}
d\left|\partial_{z} T\right|^{2}+2\left\|\partial_{z} T\right\|^{2} d t= & 2\left(\partial_{z} F_{T}, \partial_{z} T\right) d t+\left|\partial_{z} \sigma_{T}(\mathbf{v}, T, S)\right|_{L_{2}(\mathfrak{U}, H)}^{2} d t \\
& -2 \int_{\mathcal{M}} \partial_{z}\left[(\mathbf{v} \cdot \nabla) T+w(\mathbf{v}) \partial_{z} T\right] \partial_{z} T d \mathcal{M} d t+2\left(\partial_{z} \sigma_{T}(\mathbf{v}, T, S), \partial_{z} T\right) d W \\
:= & \left(J_{1}^{T}+J_{2}^{T}+J_{3}^{T}+J_{4}^{T}\right) d t+J_{5}^{T} d W
\end{aligned}
$$

For the nonlinear term, $J_{3}^{T}$, an integration by parts reveals that,

$$
-J_{3}^{T}=\sum_{j} \int_{\mathcal{M}}\left(v_{j} \partial_{j z} T+\partial_{z} v_{j} \partial_{j} T\right) \partial_{z} T d \mathcal{M}=\sum_{j} \int_{\mathcal{M}}\left(v_{j} \partial_{j z} T \partial_{z} T-\partial_{z j} v_{j} T \partial_{z} T-\partial_{z} v_{j} T \partial_{z j} T\right) d \mathcal{M},
$$

and we may therefore estimate

$$
\begin{aligned}
\left|J_{3}^{T}\right| & \leq c\left(|\mathbf{v}|_{L^{4}}\left\|\partial_{z} T\right\|\left|\partial_{z} T\right|_{L^{4}}+\left\|\partial_{z} \mathbf{v}\right\||T|_{L^{4}}\left|\partial_{z} T\right|_{L^{4}}+\left|\partial_{z} \mathbf{v}\right|_{L^{4}}|T|_{L^{4}}\left\|\partial_{z} T\right\|\right) \\
& \leq c\left(|\mathbf{v}|_{L^{4}}\left\|\partial_{z} T\right\|^{7 / 4}\left|\partial_{z} T\right|^{1 / 4}+\left\|\partial_{z} \mathbf{v}\right\||T|_{L^{4}}\left|\partial_{z} T\right|^{1 / 4}\left\|\partial_{z} T\right\|^{3 / 4}\left|\partial_{z} \mathbf{v}\right|^{1 / 4}\left\|\partial_{z} \mathbf{v}\right\|^{3 / 4}|T|_{L^{4}}\left\|\partial_{z} T\right\|\right) \\
& \leq c\left(|\mathbf{v}|_{L^{4}}^{8}\left|\partial_{z} T\right|^{2}+\left\|\partial_{z} \mathbf{v}\right\|^{2}|T|_{L^{4}}^{2}+\left\|\partial_{z} \mathbf{v}\right\|^{2}+\left|\partial_{z} \mathbf{v}\right|^{2}|T|_{L^{4}}^{8}\right)+\frac{1}{2}\left(\left|\partial_{z} T\right|^{2}+\left\|\partial_{z} T\right\|^{2}\right)
\end{aligned}
$$

Also,

$$
J_{4}^{T}=\int_{\mathcal{M}} w(\mathbf{v}) \partial_{z} T \partial_{z z} T d \mathcal{M}=-\frac{1}{2} \int_{\mathcal{M}} \partial_{z} w(\mathbf{v})\left(\partial_{z} T\right)^{2} d \mathcal{M}=\frac{1}{2} \int_{\mathcal{M}} \partial_{j} v_{j}\left(\partial_{z} T\right)^{2} d \mathcal{M}=-\int_{\mathcal{M}} v_{j} \partial_{j z} T \partial_{z} T d \mathcal{M},
$$

so that

$$
\left|J_{4}^{T}\right| \leq c|\mathbf{v}|_{L^{4}}\left\|\partial_{z} T\right\|^{7 / 4}\left|\partial_{z} T\right|^{1 / 4} \leq c|\mathbf{v}|_{L^{4}}^{8}\left|\partial_{z} T\right|^{2}+\frac{1}{2}\left\|\partial_{z} T\right\|^{2} .
$$

The remaining drift terms are estimated directly using assumption (2.13):

$$
\left|J_{1}^{T}+J_{2}^{T}\right| \leq c\left(1+\|U\|^{2}+|F|^{2}\right)+\left\|\partial_{z} T\right\|^{2} .
$$

As above, in (5.10), we estimate the terms involving $J_{5}^{T}$ with the BDG inequality. We obtain for $\tau_{a} \leq \tau_{b} \leq$ $t \wedge \sigma_{K} \wedge \xi$

$$
\mathbb{E} \sup _{t \in\left[\tau_{a}, \tau_{b}\right]}\left|\int_{\tau_{a}}^{t} J_{5} d W\right| \leq \frac{1}{2} \mathbb{E} \sup _{t \in\left[\tau_{a}, \tau_{b}\right]}\left|\partial_{z} T\right|^{2}+c \mathbb{E} \int_{\tau_{a}}^{\tau_{b}}\left(1+\|U\|^{2}\right) d t^{\prime}
$$

With the stochastic Gronwall lemma, Proposition 6.3, the estimates (5.21), (5.22), (5.23), (5.24) imply (5.17). (5.18) now follows directly from (5.17), Proposition 4.1, Corollary 5.1 and Lemma 5.1. The proof of Proposition 5.3 is thus complete.

Proof of Proposition 5.1 (conclusion). Due to Corollary 5.1 and Proposition 5.3 we infer (5.4) and (5.18) and hence (5.1). Now (5.1) implies that $\lim _{K \uparrow \infty} \tau_{K}^{(2)}=\infty$, completing the proof of the proposition. 


\section{Appendix: Auxilary Results}

We collect here, for the convenience of the reader, several technical results that have been used in an essential way in the analysis above.

\subsection{Pressure Estimates}

We have made use of a special case of [SvW86, Theorem 2.12] in the proof of Proposition 4.3. This result in [SvW86] provides $L_{t}^{s} L_{x}^{r}$ estimates for the pressure terms appearing in the linear Stokes equation.

Proposition 6.1. Suppose that $d \geq 2$ and that $\mathcal{M}_{0} \subset \mathbb{R}^{d}$ is a bounded open domain with smooth boundary. Assume that $r \in(1,2)$ and that $\mathbf{f} \in L_{\text {loc }}^{2}\left([0, \infty) ; L^{r}\left(\mathcal{M}_{0}\right)\right)$. If $(\mathbf{q}, p)$ solves the Stokes equation in $\mathcal{M}_{0} \times[0, \infty)$

$$
\partial_{t} \mathbf{q}-\nu \Delta \mathbf{q}+\nabla p=\mathbf{f}, \quad \nabla \cdot \mathbf{q}=0, \quad \mathbf{q}_{\mid \partial \mathcal{M}_{0}}=0
$$

then, for any $0 \leq \tau_{0} \leq \tau_{1}<\infty$,

$$
\int_{\tau_{0}}^{\tau_{1}}|\nabla p|_{L^{r}\left(\mathcal{M}_{0}\right)}^{2} d s \leq c\left(\left\|\mathbf{q}\left(\tau_{0}\right)\right\|^{2}+\int_{\tau_{0}}^{\tau_{1}}|\mathbf{f}|_{L^{r}\left(\mathcal{M}_{0}\right)}^{2} d s\right)
$$

where $c=c\left(d, r, \mathcal{M}_{0}\right)$ is an absolute constant independent of $\mathbf{f}, \tau_{0}, \tau_{1}$.

\subsection{Two Nonstandard Gronwall Lemmas}

We made significant use of two non-standard versions of the Gronwall Lemma in the analysis above. The first version, Proposition 6.2, is used to close the estimates for $\mathbf{v}$ in $L^{4}$ for the proof of Proposition 4.1 in Section 4. The second version, Proposition 6.3, has been useful for the analysis of stochastic evolution equation and is employed here in Theorem 3.2, Proposition 5.2, Lemma 5.1, and Proposition 5.3.

The following proposition is somehow new:

Proposition 6.2. Suppose that, for some $t \geq 0$, we are given $f, g, h \in L^{1}([0, t])$ and $X \in C([0, t))$. Assume that $X, f, g, h$ are all positive for a.e. $t^{\prime} \in[0, t]$ and that $f$ is continuous at $t^{\prime}=0$. If, for almost every $0 \leq \tau_{a} \leq \tau_{b}<t$, and for some fixed $p \geq 1$, we have

$$
\sup _{t^{\prime} \in\left[\tau_{a}, \tau_{b}\right]} X\left(t^{\prime}\right) \leq f\left(\tau_{a}\right)^{p}+\sup _{t^{\prime} \in\left[\tau_{a}, \tau_{b}\right]} X\left(t^{\prime}\right) \int_{\tau_{a}}^{\tau_{b}} g\left(t^{\prime}\right) d t^{\prime}+\int_{\tau_{a}}^{\tau_{b}} h\left(t^{\prime}\right) d t^{\prime},
$$

then there exists an absolute constant $c=c\left(t, p,|f(0)|,|f|_{L^{1}},|g|_{L^{1}}\right)$ such that,

$$
\sup _{t^{\prime} \in[0, t]} X\left(t^{\prime}\right) \leq c\left(1+\int_{0}^{t} h\left(t^{\prime}\right) d t^{\prime}\right)
$$

Proof. We begin with some preliminaries to determine the constant $c$ in (6.3). Choose $\epsilon>0$ so that $\int_{s^{\prime}}^{s} g\left(t^{\prime}\right) d t^{\prime}<1 / 2$ for any pair $0 \leq s^{\prime} \leq s \leq t$ such that $s-s^{\prime}<\epsilon$. Now choose $n$ in such a way that $t / n<\epsilon / 2$. Then pick $M>\max \{2,|f(0)|\}$ large enough such that $\lambda(|f| \geq M)<t /(4 n)$, where $\lambda$ is the Lebesgue measure on the interval $[0, t]$. This later quantity $M$ exists as a consequence of the Chebshev inequality and the fact that $f \in L^{1}([0, t])$. We now show $(6.3)$ is satisfied by taking $c=2 M^{p}$.

To this end fix any $t^{*} \in(t / 2, t)$. Set $t_{0}=0, t_{n}=t^{*}$. For each $k=1, \ldots, n-1$ we may pick an element $t_{k} \in\left(t^{*} k / n, t^{*}(k+1) / n\right)$ so that $f\left(t_{k}\right)<M$. Note also that according to this choice $t_{k+1}-t_{k}<\epsilon$. Thus, for $k=0,1, \ldots, n$, by applying $(6.2)$ with $\tau_{a}=t_{k}, \tau_{b}=t_{k+1}$, we infer,

$$
\sup _{t^{\prime} \in\left[t_{k}, t_{k+1}\right]} X\left(t^{\prime}\right) \leq M^{p}+\frac{1}{2} \sup _{t^{\prime} \in\left[t_{k}, t_{k+1}\right]} X\left(t^{\prime}\right)+\int_{t_{k}}^{t_{k+1}} h\left(t^{\prime}\right) d t^{\prime} .
$$


Noting that $X \in C([0, t)$, we may rearrange and infer:

$$
\sup _{t^{\prime} \in\left[t_{k}, t_{k+1}\right]} X\left(t^{\prime}\right) \leq 2 M^{p}+2 \int_{t_{k}}^{t_{k+1}} h\left(t^{\prime}\right) d t^{\prime} \leq 2 M^{p}\left(1+\int_{t_{k}}^{t_{k+1}} h\left(t^{\prime}\right) d t^{\prime}\right) .
$$

The analogue of (6.3), with $t^{*}$ replacing $t$ on the left hand side of the inequality now follows from a simple induction. Since $M$ maybe chosen independently of $t^{*} \in(t / 2, t)$ the proof is now complete.

The following 'stochastic Gronwall Lemma' has been established in [GHZ09]:

Proposition 6.3. Fix $t>0$ and assume that $X, Y, Z, R:[0, t) \times \Omega \rightarrow \mathbb{R}$ are real valued, non-negative stochastic processes. Let $\tau<t$ be a stopping time so that

$$
\mathbb{E} \int_{0}^{\tau}(R X+Z) d s<\infty
$$

Assume, moreover that for some fixed constant $\kappa$

$$
\int_{0}^{\tau} R d s<\kappa, \quad \text { a.s. }
$$

Suppose that for all stopping times $0 \leq \tau_{a}<\tau_{b} \leq \tau$

$$
\mathbb{E}\left(\sup _{t \in\left[\tau_{a}, \tau_{b}\right]} X+\int_{\tau_{a}}^{\tau_{b}} Y d s\right) \leq c_{0} \mathbb{E}\left(X\left(\tau_{a}\right)+\int_{\tau_{a}}^{\tau_{b}}(R X+Z) d s\right)
$$

where $c_{0}$ is a constant independent of the choice of $\tau_{a}, \tau_{b}$. Then

$$
\mathbb{E}\left(\sup _{t \in[0, \tau]} X+\int_{0}^{\tau} Y d s\right) \leq c \mathbb{E}\left(X(0)+\int_{0}^{\tau} Z d s\right),
$$

where $c=c\left(c_{0}, t, \kappa\right)$.

\section{Acknowledgments}

This work was partially supported by the National Science Foundation (NSF) under the grants DMS-1004638, DMS-0906440 and by the Research Fund of Indiana University. We thank M. Petcu for her helpful comments and suggestions.

\section{References}

[BF00] A. Bensoussan and J. Frehse. Local solutions for stochastic Navier Stokes equations. M2AN Math. Model. Numer. Anal., 34(2):241-273, 2000. Special issue for R. Temam's 60th birthday.

[Bje04] V. Bjerknes. Das problem der wettervorhersage, betrachtet vom standpunkte der mechanik und der physik. Meteorol. Z., 21:1-7, 1904.

[BP00] Z. Brzeźniak and S. Peszat. Strong local and global solutions for stochastic Navier-Stokes equations. In Infinite dimensional stochastic analysis (Amsterdam, 1999), volume 52 of Verh. Afd. Natuurkd. 1. Reeks. K. Ned. Akad. Wet., pages 85-98. R. Neth. Acad. Arts Sci., Amsterdam, 2000 .

[Bre00] H. Breckner. Galerkin approximation and the strong solution of the Navier-Stokes equation. J. Appl. Math. Stochastic Anal., 13(3):239-259, 2000. 
[BSLP09] J. Berner, G. J. Shutts, M. Leutbecher, and T. N. Palmer. A spectral stochastic kinetic energy backscatter scheme and its impact on flow-dependent predictability in the ecmwf ensemble prediction system. Journal of the Atmospheric Sciences, 66(3):603-626, 2009.

[BT73] A. Bensoussan and R. Temam. Équations stochastiques du type Navier-Stokes. J. Functional Analysis, 13:195-222, 1973.

[CG94] M. Capiński and D. Gatarek. Stochastic equations in Hilbert space with application to NavierStokes equations in any dimension. J. Funct. Anal., 126(1):26-35, 1994.

[Cru89] A. B. Cruzeiro. Solutions et mesures invariantes pour des équations d'évolution stochastiques du type Navier-Stokes. Exposition. Math., 7(1):73-82, 1989.

[CT07] C. Cao and E. Titi. Global well-posedness of the three-dimensional viscous primitive equations of large scale ocean and atmosphere dynamics. Ann. of Math. (2), 166(1):245-267, 2007.

[DGHT11] A. Debussche, N. Glatt-Holtz, and R. Temam. Local martingale and pathwise solutions for an abstract fluids model. Physica D, 2011. (to appear).

[DPD03] G. Da Prato and A. Debussche. Ergodicity for the 3D stochastic Navier-Stokes equations. $J$. Math. Pures Appl. (9), 82(8):877-947, 2003.

[DPZ92] G. Da Prato and J. Zabczyk. Stochastic equations in infinite dimensions, volume 44 of Encyclopedia of Mathematics and its Applications. Cambridge University Press, Cambridge, 1992.

[DPZ96] G. Da Prato and J. Zabczyk. Ergodicity for infinite-dimensional systems, volume 229 of London Mathematical Society Lecture Note Series. Cambridge University Press, Cambridge, 1996.

[EPT07] B. Ewald, M. Petcu, and R. Temam. Stochastic solutions of the two-dimensional primitive equations of the ocean and atmosphere with an additive noise. Anal. Appl. (Singap.), 5(2):183$198,2007$.

[ET99] I. Ekeland and R. Temam. Convex analysis and variational problems, volume 28 of Classics in Applied Mathematics. Society for Industrial and Applied Mathematics (SIAM), Philadelphia, PA, english edition, 1999. Translated from the French.

[FG95] F. Flandoli and D. Gatarek. Martingale and stationary solutions for stochastic Navier-Stokes equations. Probab. Theory Related Fields, 102(3):367-391, 1995.

[FR02] Franco Flandoli and Marco Romito. Partial regularity for the stochastic Navier-Stokes equations. Trans. Amer. Math. Soc., 354(6):2207-2241 (electronic), 2002.

[FR08] F. Flandoli and M. Romito. Markov selections for the 3D stochastic Navier-Stokes equations. Probab. Theory Related Fields, 140(3-4):407-458, 2008.

[GGMRB01] F. Guillén-González, N. Masmoudi, and M. A. Rodríguez-Bellido. Anisotropic estimates and strong solutions of the primitive equations. Differential Integral Equations, 14(11):1381-1408, 2001.

[GH09] B. Guo and D. Huang. 3d stochastic primitive equations of the large-scale ocean: global well-posedness and attractors. Commun. Math. Phys., 286:697-723, 2009.

[GHT11a] N. Glatt-Holtz and R. Temam. Cauchy convergence schemes for some nonlinear partial differential equations. Applicable Analysis, 90(1):85 - 102, 2011.

[GHT11b] N. Glatt-Holtz and R. Temam. Pathwise solutions of the 2-d stochastic primitive equations. Applied Mathematics and Optimization, 63(3):401-433(33), June 2011. 
[GHTT] N. Glatt-Holtz, R. Temam, and J. Tribbia. Some remarks on the role of stochastic parameterization in the equations of the ocean and atmosphere. (manuscript in preparation).

[GHZ08] N. Glatt-Holtz and M. Ziane. The stochastic primitive equations in two space dimensions with multiplicative noise. Discrete Contin. Dyn. Syst. Ser. B, 10(4):801-822, 2008.

[GHZ09] N. Glatt-Holtz and M. Ziane. Strong pathwise solutions of the stochastic Navier-Stokes system. Advances in Differential Equations, 14(5-6):567-600, 2009.

[GK96] I. Gyöngy and N. Krylov. Existence of strong solutions for Itô's stochastic equations via approximations. Probab. Theory Related Fields, 105(2):143-158, 1996.

[HTZ03] C. Hu, R. Temam, and M. Ziane. The primitive equations on the large scale ocean under the small depth hypothesis. Discrete Contin. Dyn. Syst., 9(1):97-131, 2003.

[Kob06] G. M. Kobelkov. Existence of a solution 'in the large' for the 3D large-scale ocean dynamics equations. C. R. Math. Acad. Sci. Paris, 343(4):283-286, 2006.

[Kob07] G. M. Kobelkov. Existence of a solution "in the large" for ocean dynamics equations. J. Math. Fluid Mech., 9(4):588-610, 2007.

[Kry99] N. V. Krylov. An analytic approach to SPDEs. In Stochastic partial differential equations: six perspectives, volume 64 of Math. Surveys Monogr., pages 185-242. Amer. Math. Soc., Providence, RI, 1999.

[KZ07] I. Kukavica and M. Ziane. On the regularity of the primitive equations of the ocean. Nonlinearity, 20(12):2739-2753, 2007.

[LL79] D. C. Leslie and Quarini G. L. The application of turbulence theory to the formulation of subgrid modelling procedures. Journal of Fluid Mechanics, 91:65-91, 1979.

[LTW92a] J.-L. Lions, R. Temam, and S. H. Wang. New formulations of the primitive equations of atmosphere and applications. Nonlinearity, 5(2):237-288, 1992.

[LTW92b] J.-L. Lions, R. Temam, and S. H. Wang. On the equations of the large-scale ocean. Nonlinearity, 5(5):1007-1053, 1992.

[LTW93] J.-L. Lions, R. Temam, and S. Wang. Models for the coupled atmosphere and ocean. (CAO I,II). Comput. Mech. Adv., 1(1):120, 1993.

[MR01] R. Mikulevicius and B. Rozovskii. A note on Krylov's $L_{p}$-theory for systems of SPDEs. Electron. J. Probab., 6:no. 12, 35 pp. (electronic), 2001.

[MR04] R. Mikulevicius and B. L. Rozovskii. Stochastic Navier-Stokes equations for turbulent flows. SIAM J. Math. Anal., 35(5):1250-1310, 2004.

[MR05] R. Mikulevicius and B. L. Rozovskii. Global $L_{2}$-solutions of stochastic Navier-Stokes equations. Ann. Probab., 33(1):137-176, 2005.

[MT92] P. J. Mason and D. J. Thomson. Stochastic backscatter in large-eddy simulations of boundary layers. Journal of Fluid Mechanics, 242:51-78, 1992.

[Ped82] J. Pedlosky. Geophysical Fluid Dynamics. Springer Verlag, 1982.

[Pol71] E. Polak. Computational methods in optimization. A unified approach. Mathematics in Science and Engineering, Vol. 77. Academic Press, New York, 1971. 
[Pol97] E. Polak. Optimization, volume 124 of Applied Mathematical Sciences. Springer-Verlag, New York, 1997. Algorithms and consistent approximations.

[PR07] C. Prévôt and M. Röckner. A concise course on stochastic partial differential equations, volume 1905 of Lecture Notes in Mathematics. Springer, Berlin, 2007.

[PTZ08] M. Petcu, R. Temam, and M. Ziane. Some mathematical problems in geophysical fluid dynamics. In Special Volume on Computational Methods for the Atmosphere and the Oceans, volume 14 of Handbook of Numerical Analysis, pages 577-750. Elsevier, 2008.

[Ric07] Lewis F. Richardson. Weather prediction by numerical process. Cambridge Mathematical Library. Cambridge University Press, Cambridge, second edition, 2007. With a foreword by Peter Lynch.

[Roc70] R. Tyrrell Rockafellar. Convex analysis. Princeton Mathematical Series, No. 28. Princeton University Press, Princeton, N.J., 1970.

[Ros77] H. A. Rose. Eddy diffusivity, eddy noise and subgrid-scale modelling. Journal of Fluid Mechanics, 81:719-734, 1977.

[RTT08] A. Rousseau, R. Temam, and J. Tribbia. Boundary value problems for the inviscid primitive equations in limited domain. In Special Volume on Computational Methods for the Atmosphere and the Oceans, volume 14 of Handbook of Numerical Analysis, pages 577-750. Elsevier, 2008.

[Shi07] Armen Shirikyan. Qualitative properties of stationary measures for three-dimensional NavierStokes equations. J. Funct. Anal., 249(2):284-306, 2007.

[SvW86] Hermann Sohr and Wolf von Wahl. On the regularity of the pressure of weak solutions of Navier-Stokes equations. Arch. Math. (Basel), 46(5):428-439, 1986.

[Vio76] M. Viot. Solutions faibles d'équations aux dérivées partielles non linéaires. $1976 . \quad$ Thèse, Université Pierre et Marie Curie, Paris.

[ZF10] M. J. Zidikheri and J. S. Frederiksen. Stochastic subgrid-scale modelling for non-equilibrium geophysical flows. Philosophical Transactions of the Royal Society A: Mathematical, Physical and Engineering Sciences, 368(1910):145-160, 2010.

[Zia95] M. Ziane. Regularity results for Stokes type systems. Appl. Anal., 58(3-4):263-292, 1995. 
Arnaud Debussche

Département de Mathématiques

ENS Cachan Bretagne

Web: http://w3.bretagne.ens-cachan.fr/math/people/arnaud.debussche/

Email: arnaud.debussche@bretagne.ens-cachan.fr

\section{Nathan Glatt-Holtz}

Department of Mathematics

Indiana University

Web: http://mypage.iu.edu/ negh/

Email: negh@indiana.edu

\section{Roger Temam}

Department of Mathematics

Indiana University

Web: http://mypage.iu.edu/ temam/

Email: temam@indiana.edu

Mohammed Ziane

Department of Mathematics

University of Southern California

Web: http://www-bcf.usc.edu/ ziane/

Email: ziane@usc.edu 